\title{
Global decline in aggregated migrations of large terrestrial mammals
}

\author{
Grant Harris $^{1,2, *}$, Simon Thirgood ${ }^{3,4}$, J. Grant C. Hopcraft ${ }^{3,5}$, \\ Joris P. G. M. Cromsigt ${ }^{6}$, Joel Berger ${ }^{7,8}$ \\ ${ }^{1}$ Center for Biodiversity and Conservation, American Museum of Natural History, Central Park West, 79th Street, \\ New York, New York 10024, USA \\ ${ }^{2}$ US Fish \& Wildlife Service, PO Box 1306, Albuquerque, New Mexico 87103, USA \\ ${ }^{3}$ Frankfurt Zoological Society, PO Box 14935, Arusha, Tanzania \\ ${ }^{4}$ Aberdeen Centre for Environmental Sustainability, Macaulay Institute, Aberdeen AB34 5EL, UK \\ ${ }^{5}$ Community and Conservation Ecology, University of Groningen, Postbus 72, 9700 AB Groningen, The Netherlands \\ ${ }^{6}$ Mammal Research Institute, Polish Academy of Sciences, 17-230 Białowieža, Poland \\ ${ }^{7}$ Northern Rockies Field Office, Wildlife Conservation Society, University of Montana, Missoula, Montana 59812, USA \\ ${ }^{8}$ Division of Biological Sciences (HS 4824), University of Montana, Missoula, Montana 59812, USA
}

\begin{abstract}
Knowledge of mammal migrations is low, and human impacts on migrations high. This jeopardizes efforts to conserve terrestrial migrations. To aid the conservation of these migrations, we synthesized information worldwide, describing 24 large-bodied ungulates that migrate in aggregations. This synthesis includes maps of extinct and extant migrations, numbers of migrants, summaries of ecological drivers and threats migrants confront. As data are often lacking, we outlined steps for science to address and inform conservation actions. We evaluated migrants against this framework, and reported their status. Mass migrations for 6 species are extinct or unknown. Most remaining migrants $(\mathrm{n}=9$ ) occur from 6 locations in Africa, with Eurasia and North America containing 6 and 4 remaining mass migrants, respectively (with caribou/reindeer Rangifer tarandus occurring in both regions). All migrants declined in abundance, except wildebeest and other migrants in the SerengetiMara Ecosystem (SME), white-eared kob and tiang in Sudan, and some caribou populations. Protected areas only contain migrations for 5 species in the SME, chiru on the Tibetan Plateau, and some caribou populations in North America. Most mass migrants track the seasonal and shifting patterns of greening vegetation over expanses of savannahs, steppes, and grasslands. Principal threats include overhunting and habitat loss from livestock, agriculture, and fencing that excludes animals from forage or water. Conservation science overlooks numerous migrations, so many have already disappeared and continue to do so. Key principles for conserving migrants, exemplified by the SME and Greater Yellowstone Ecosystem (GYE), include securing seasonal ranges, resource protection, government support and minimizing fences. This review forms a baseline for initiating conservation action for many ungulate migrations needing attention.
\end{abstract}

KEY WORDS: Mammals · Migration · Aggregation · Serengeti-Mara • Yellowstone · Ungulate · Global audit Resale or republication not permitted without written consent of the publisher

\section{INTRODUCTION}

Migration, the seasonal and round-trip movement of animals between discrete areas (Berger 2004, Thirgood et al. 2004), is a behavior common to a diversity of taxa (Dingle 1996). A few migrations are well known, such as the movements made by 1.3 million wildebeest Connochaetes taurinus in the Serengeti-Mara Ecosystem (SME) of Tanzania and Kenya (Thirgood et al. 2004). Other migrations are obscure, such as those car- 
ried out by eland Tragelaphus oryx in Botswana (C. Thouless unpubl. data, R. Brett pers. comm.). Overall, our knowledge of migrations is low (Berger 2004), and human impact high (Vitousek et al. 1986, Pimm et al. 2001); this jeopardizes the conservation of many migratory species (Berger 2004).

Conservationists worry about the persistence of migrations (Wilcove \& Wikelski 2008). Some issues are ecological, as mass migrants have positive feedback effects on grassland forage and indirect effects on ecosystem processes (e.g. increasing grassland production and raising nitrogen mineralization) (Caughley 1976, McNaughton et al. 1988, Frank 1998), and therefore losing migrations may result in ecosystem collapse. Economics also plays a part. For example, were there no wildebeest migration in the SME, herbivore populations would almost certainly decline, carnivore populations would follow, and Serengeti would lose tourism dollars. Without financing, resource protection would slacken and the natural ecosystem would disintegrate, as livestock, agriculture and other developments replaced it. Ethical issues are also involved. Eradicating migrations and relegating migrants to zoos or fenced parks represents one of the worst examples of destructive human impact. Their senseless destruction by a shortsighted few causes long-term losses in the natural spectacles for many. Humanity can and should advance society while maintaining such migrations.

Unfortunately, conservationists are largely unaware of which migrations are lost, which ones remain, the factors threatening the remainder, and tangible management solutions to protect the phenomenon. Consequently, we audited 24 large mammals that migrate or migrated in aggregations to evaluate their conservation status. Our efforts involved 6 components: (1) Preserving migrations means knowing where they remain and where they have been lost. Therefore, we mapped locations for extant and extinct aggregated migrations. (2) We summarized attributes pertinent for their conservation, namely the number of animals migrating and the distances traveled. (3) Conserving migrations requires knowing why they occur, so we examined ecological drivers. Site-specific studies of migratory ungulates identify the importance of gradients in rainfall, forage growth, and nutrient availability in driving seasonal migrations (Fryxell \& Sinclair 1988a, Murray 1995, Boone et al. 2006). We investigated whether such resource gradients are widespread phenomena in migratory ecosystems. (4) We synthesized threats facing these migrations, examined their commonalities, and reported their consequences. (5) We evaluated all sources in a 6-step framework and reported the state of knowledge necessary to conserve these migratory species. (6) We described actions necessary to sustain mass migrations by exemplifying successes learned elsewhere. Overall, we provide a baseline for guiding and catalyzing conservation action for many migrations needing attention.

\section{MATERIALS AND METHODS}

We concentrated on terrestrial mammals with body mass $>20 \mathrm{~kg}$ (Morrison et al. 2007). We then determined which species (including subspecies) are, or once were, migratory in aggregated masses. We defined an 'aggregation' as hundreds to thousands of animals that move or once moved in masses. We do not specify a clear threshold for group size or migratory distances. When mass migrations decline, the numbers of migrants and the distances traveled often fall. Quantifying a threshold requires credible historic and current data, which is lacking for most species and systems.

Given that most species have not been studied with respect to migration per se, we began with 'Walkers Mammals of the World' (Nowak 1999), with supplementary information for 20 African species (Estes 1991). Despite their shortcomings, these sources review the distribution, population status and natural history of these species. We used them to list mammals that migrate in aggregations, once migrated, or lack concrete data but for which natural history suggested potentially migratory behavior. For these species, we then examined scientific literature to determine where (or if) aggregated migrations remain, and information describing their attributes. Searches used 'Web of Science' and 'Google Scholar' engines, with terms including the species common name and scientific name alone or in combination with the word 'migration'. We also consulted gray literature, websites, scientists and game managers familiar with target species. From these sources, we digitized migratory areas based on their geographical locations, and summarized attributes, ecological drivers and threats for each migratory species.

Conserving mass migrations requires descriptive data, information to evaluate their status, and collaborations to foster their protection. This process forms a $6-$ step framework: (1) Mapping species movements to determine the locations that migrants go, and the routes taken to get there. (2) Determining migrants' habitat needs, namely seasonal ranges and transition areas between these ranges. (3) Mapping migratory habitats to determine their geographical location, and evaluating how much remains. (4) Identifying the factors threatening the sustainability of migratory habitats (or the migrants themselves), and where these threats are or are likely to occur. (5) Determining how much of the migratory route and migratory habitat is required to maintain 
a given population objective to ensure the sustainability of the mass migration. This should account for neighboring land uses and threats to routes or habitat. (6) Work with partners, wildlife managers, policy makers and opposing interest groups to better manage and conserve mass migrants, routes and habitat.

While these steps generally progress with increasing knowledge and details on migrations, some can be taken before completing previous steps. For example, Step 4 may happen without full knowledge covering Steps 1 to 3 . Ideally, all 6 steps require scientific approaches, and if applicable, results should be presented in peer-reviewed literature. Steps 1 to 5 are relatively consistent between migratory systems, while Step 6, which seeks solutions, will likely vary depending on the species, situation and site.

We evaluated available sources describing mass migrants against this framework. Values represent our interpretation and we present them as relative measures. Results illustrate the state of information pertinent for conserving these migratory species.

\section{RESULTS}

\section{Global audit}

Twenty-four large mammal species (and subspecies) are known to migrate or to have migrated in aggregations - all ungulates. Mass migrations for 6 of these are extinct or their status unknown: springbok Antidorcas marsupialis, black wildebeest Connochaetes gnou, blesbok Damaliscus dorcas, kulan Equus hemionus, scimitar horned oryx Oryx dammah, quagga Equus quagga. Most migratory populations lack reports on their numbers, distances traveled, geographical routes, ecological drivers and threats. Where data exist, they are often over a decade old (Tables 1 \& 2).

We plotted the geographical locations where aggregated migrations remain and, when documented, those lost (Figs. $1 \& 2$ ). Most migrants are African ( $\mathrm{n}=$ 14). Migrations for the 9 remaining migrants occur in just 6 locations (Boma-Jonglei, Sudan; SerengetiMara Ecosystem, Tanzania/Kenya; Tarangire, Tanzania; Liuwa, Zambia/Angola; Chobe, Botswana and; Kalihari, Botswana) (Fig. 1). Protected areas in Africa only enclosed migrations within the SME. There are reports on 7 aggregated migrants (6 with migrations remaining) for Eurasia, and 4 for North America, with caribou/ reindeer Rangifer tarandus in both (Tables 1 \& 2). Protected areas largely contain migrations of chiru Pantholopos hodgsoni on the Tibetan Plateau and some caribou populations in North America (Fig. 2). South America and Australia lack reports of mass migrations.

\section{Ecological drivers}

The ecological drivers of mass migrations and the threats that migrations confront are connected, as threats disable the drivers. Hence, we must first understand why mass migrations occur, in order to identify the threats, appreciate how they work, and arm ourselves to alleviate or pre-empt them. We identified 4 dominant factors driving mass migrations: seasonal availability of forage (quality/quantity), snow depth, use of traditional areas, and surface water availability. Forage moisture, a fifth factor related to forage quality, was only specifically documented for pronghorn Antilocarpa americana.

Migrants move from locations where food quality and quantity is poor or inaccessible to places where it is more abundant, nutritious and available. Most migrants seek young grass, because this is most digestible and high in protein (Hanley 1982). The quality and quantity of grass depends on the availability of water (rain in tropical and temperate savannas and grasslands, snowmelt in northern mountains and plains), which varies in timing, amount, and distribution across these species' ranges (Deshmukh 1984, Williamson et al. 1988). Animals track the seasonal and shifting distribution of their forage ('green flushes') and therefore become migratory (our Table 2, McNaughton 1985, Fryxell \& Sinclair 1988a, Morgantini \& Hudson 1988, Murray 1995, Boone et al. 2006, Mueller et al. 2008). This driver explains the movements of 17 migrants. When rainfall is the determining factor, this type of migration occurs in all of the African migrants for which we have data, and also for saiga Saiga tatarica, Mongolian gazelle Procapra gutturosa, kulan and pronghorn. Snowmelt across elevation gradients and the resulting vegetation response influences the movements of bison Bison bison, elk Cervus elaphus and caribou. Early references describe this qualitatively, with recent material quantifying the response (Table 2).

Deep snow obstructs migrants' access to forage in winter months. This driver affects migratory patterns in all of the North American and Eurasian migrants, by forcing them to move toward lower elevations or latitudes (Table 2). As above, migrants reverse movements during snowmelts, to capitalize on greening flushes of vegetation.

Changes in resource availability can be predictable or unpredictable, resulting in different migratory responses. The distribution of snow across elevations and interior (continental) regions is relatively predictable. Animals can conform to this regularity and become habituated to areas where forage is reliable over time. Hence, half of the northern migrants use traditional routes and ranges, often spanning generations 
Table 1. Summary of historic and current aggregated migrations for terrestrial mammals, including distances traveled, numbers of migrants, and sources reporting non-migratory populations. The column, 'Mass migration', shows the location of migrants in Figs. 1 \& 2 (no. in bold before literature source). Literature sources and geographical locations of migrations (in parentheses) given after

\begin{tabular}{|c|c|c|c|c|}
\hline \multirow{2}{*}{$\begin{array}{l}\text { Scientific } \\
\text { name }\end{array}$} & \multirow{2}{*}{$\begin{array}{l}\text { Common } \\
\text { name }\end{array}$} & \multirow[t]{2}{*}{ Location } & \multicolumn{2}{|c|}{ Mass migration } \\
\hline & & & Current & Historic \\
\hline $\begin{array}{l}\text { Alcelaphus } \\
\text { buselaphus }\end{array}$ & Hartebeest & Africa & $\begin{array}{l}\text { 1 - Skinner \& Smithers (1990) (Botswana) }{ }^{*}, \text { Estes } \\
(1991)^{*}, \mathbf{2} \text { - Verlinden (1998) (Kalahari, Botswana), } \\
\mathbf{3} \text { - (1) (Kalahari, Botswana) }{ }^{*}\end{array}$ & $\begin{array}{l}4 \text { - Swayne (1894) (Somalia), } 5 \text { - Foster \& } \\
\text { Kearney (1967) (Nairobi, Kenya), } 6 \text { - (2) } \\
\text { (Tsavo, Kenya) }{ }^{*}, \text { Skinner \& Smithers } \\
(1990) \text { (Botswana) }{ }^{*},(1) \text { (Kalahari, Bots- } \\
\text { wana) }^{*}, \mathbf{7}-(2) \text { (Voi-Tsavo-Athi, Kenya) }{ }^{*}\end{array}$ \\
\hline $\begin{array}{l}\text { Antidorcas } \\
\text { marsupialis }\end{array}$ & Springbok & Africa & 8 - Skinner (1993) (Botswana (?) & $\begin{array}{l}\text { Child \& LeRiche (1969) (Botswana), } \\
\text { Skinner (1993) (Namibia, South Africa), } \\
\text { Skinner \& Louw (1996) (Botswana, } \\
\text { Namibia, South Africa) }^{*}, \text { Skinner \& } \\
\text { Chimimba (2005) (South Africa) }^{*}\end{array}$ \\
\hline $\begin{array}{l}\text { Conno- } \\
\text { chaetes } \\
\text { gnou }\end{array}$ & $\begin{array}{l}\text { Black } \\
\text { wildebeest }\end{array}$ & Africa & & $\begin{array}{l}9 \text { - Estes (1991) (South Africa) }{ }^{*}, \\
10 \text { - Skinner \& Smithers (1990) (South } \\
\text { Africa) }{ }^{*}, \text { Estes (1991) (South Africa) }{ }^{*}, \\
\text { Skinner (1993), Skinner \& Chimimba }_{(2005) \text { (South Africa) }^{*}}\end{array}$ \\
\hline $\begin{array}{l}\text { Conno- } \\
\text { chaetes } \\
\text { taurinus }\end{array}$ & $\begin{array}{l}\text { Blue } \\
\text { wildebeest }\end{array}$ & Africa & 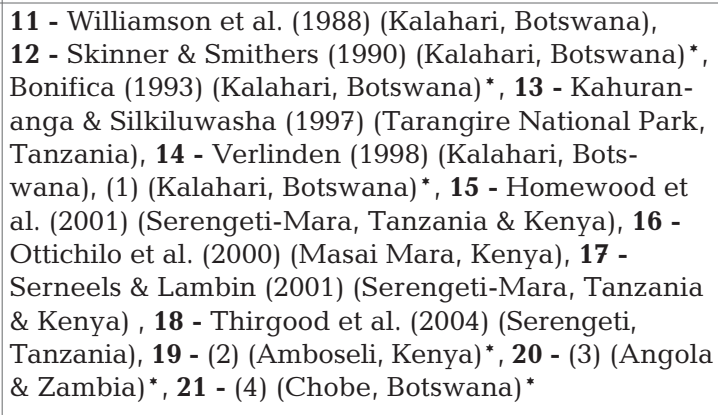 & $\begin{array}{l}\text { 22 - Berry (1997) (Etosha, Namibia), } 23 \text { - } \\
\text { Foster \& Kearney (1967) (Nairobi, Kenya), } \\
\text { Sinclair \& Norton-Griffiths (1979) } \\
\text { (Serengeti, Tanzania) }{ }^{*}, \text { Borner (1985) } \\
\text { (Tarangire, Tanzania), 24 - Whyte \& } \\
\text { Joubert (1988) (Kruger, South Africa), } \\
\text { Gasaway et al. (1996) (Etosha, Namibia), } \\
\text { Thirgood et al. (2004), (2) (Amboseli - } \\
\text { Nairobi) }^{*}, 25 \text { - (5) (Voi-Tsavo-Athi) }{ }^{*},\end{array}$ \\
\hline $\begin{array}{l}\text { Damaliscus } \\
\text { dorcas }\end{array}$ & Blesbok & Africa & & $\begin{array}{l}26 \text { - Estes (1991) (South Africa) }{ }^{*}, \text { Skinner } \\
\text { \& Smithers (1990) (South Africa) }{ }^{*}, \text { Estes } \\
(1991) \text { (South Africa) }{ }^{*}, \text { Skinner \& Chim- } \\
\text { imba (2005) (South Africa) }{ }^{*}\end{array}$ \\
\hline $\begin{array}{l}\text { Damaliscus } \\
\text { lunatus }\end{array}$ & Tiang (topi) & Africa & 27 - East $(1999)^{*},(6)$ (Boma-Jonglei, Sudan) ${ }^{*}$ & East $(1988)^{*}$ \\
\hline $\begin{array}{l}\text { Equus } \\
\text { burchellii }\end{array}$ & $\begin{array}{l}\text { Burchell's } \\
\text { zebra }\end{array}$ & Africa & 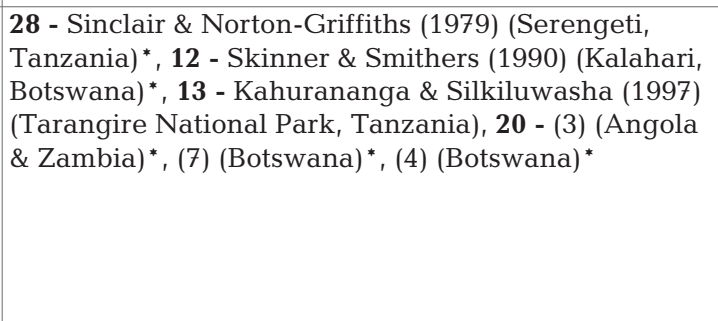 & 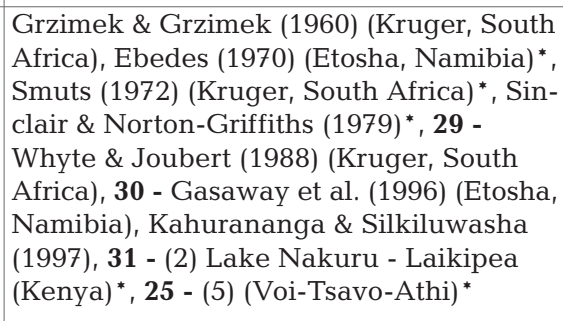 \\
\hline $\begin{array}{l}\text { Equus } \\
\text { quagga } \\
\text { quagga }\end{array}$ & Quagga & Africa & & $\begin{array}{l}32 \text { - Estes (1991) (South Africa) }{ }^{*}, \text { Estes } \\
\text { (1991) (South Africa) }{ }^{*}, \text { Skinner \& Chim- } \\
\text { imba (2005) (South Africa) }{ }^{*}\end{array}$ \\
\hline $\begin{array}{l}\text { Gazella } \\
\text { thomsonii \& } \\
\text { G. } t \text {. } \\
\text { albonotata }\end{array}$ & $\begin{array}{l}\text { Thompson's } \\
\text { gazelle \& } \\
\text { Mongalla } \\
\text { gazelle }\end{array}$ & Africa & $\begin{array}{l}33 \text { - Campbell \& Borner (1995) (Serengeti-Mara, } \\
\text { Tanzania \& Kenya) }{ }^{*} \text {, East (1999) (Boma-Jonglei, } \\
\text { Sudan) }^{*}, 27-(6) \text { (Boma-Jonglei, Sudan) }^{*}\end{array}$ & 34 - (2) Lake Nakuru - Laikipea (Kenya) ${ }^{*}$ \\
\hline Kobus kob & $\begin{array}{l}\text { White-eared } \\
\text { kob }\end{array}$ & Africa & $\begin{array}{l}35 \text { - East (1999) (Boma-Jonglei, Sudan) }{ }^{*}, 27 \text { - (6) } \\
\text { (Boma-Jonglei, Sudan) }^{*}\end{array}$ & Fryxell \& Sinclair (1988b) \\
\hline $\begin{array}{l}\text { Nanger } \\
\text { granti }\end{array}$ & $\begin{array}{l}\text { Grant's } \\
\text { gazelle }\end{array}$ & Africa & $\begin{array}{l}28 \text { - Sinclair \& Norton-Griffiths (1979) (Serengeti, } \\
{\text { Tanzania) }{ }^{*}, \text { Estes (1991) (Serengeti, Tanzania)* }}^{*}\end{array}$ & $\begin{array}{l}\text { Walther (1972) (Serengeti, Tanzania), } \\
\text { Sinclair \& Norton-Griffiths (1979) } \\
\text { (Serengeti, Tanzania) }^{*}\end{array}$ \\
\hline
\end{tabular}


sources. The numbers 1 to 18 in parentheses indicate an unpublished source, listed as a footnote to the table. An asterisk indicates literature which may not have undergone peer review

\begin{tabular}{|c|c|c|c|c|}
\hline \multirow{2}{*}{$\begin{array}{l}\text { Current migration dis- } \\
\text { tance (round trip, km) }\end{array}$} & \multirow{2}{*}{$\begin{array}{l}\text { Historic migration dis- } \\
\text { tance (round trip, km) }\end{array}$} & \multicolumn{2}{|r|}{ Numbers migrating } & \multirow{2}{*}{$\begin{array}{l}\text { Non-migratory } \\
\text { reference }\end{array}$} \\
\hline & & Current & Historic & \\
\hline $\begin{array}{l}\text { Inferred } \sim 400 \text { (Skinner }^{*} \\
\& \text { Smithers } 1990)^{*}\end{array}$ & $?$ & $\begin{array}{l}\sim 40000 \text { (Botswana, } \\
\text { Verlinden 1998) } \\
\sim 34000(1)^{*}\end{array}$ & $\begin{array}{l}\text { 1000s (Eastern \& Southern Africa) } \\
\text { Swayne (1894), } 10000+\text { (Botswana, } \\
\text { Smithers 1971) }\end{array}$ & $\begin{array}{l}\text { Estes }(1991)^{*}, \text { Murray } \\
\text { \& Brown (1993) }\end{array}$ \\
\hline $\begin{array}{l}\text { 20. Presently, most } \\
\text { likely } 0 \text { (Skinner 1993) }\end{array}$ & ? & $\begin{array}{l}\text { Cited in Skinner (1993) } \\
(2000-3000 \text { [Namibia] } \\
\text { Wakefield 1988), one } \\
600 \text { (if it remains) } \\
\text { (Skinner 1993) }\end{array}$ & $\begin{array}{l}15000 \text { (Kalahari) Child \& le Riche } \\
\text { (1969). Following cited in Skinner } \\
\text { (1993): } 100000 \text { (Karoo) Thompson } \\
\text { (1827), 5000-15000 (Karoo) Barrow } \\
(1804), 40000 \text { (Kalahari) Livingstone } \\
(1857)\end{array}$ & $\begin{array}{l}\text { Skinner (1993), } \\
\text { Gasway et al. (1996) }\end{array}$ \\
\hline 0 (Estes 1991)* $^{*}$ & $?$ & 0 (Estes 1991)* $^{*}$ & $\begin{array}{l}100 \text { 000s - Skinner \& Smithers (1990), } \\
\text { 1000s - South Africa's Karoo (Skinner } \\
1993)\end{array}$ & Estes $(1991)^{*}$ \\
\hline $\begin{array}{l}\text { 200-600 (Verlinden } \\
\text { 1998), 600 (calculated } \\
\text { from Thirgood et al. } \\
\text { 2004), } 400(3)^{*}, \sim 200 \\
\text { (Sinclair \& Arcese } 1995 \\
\text { - Serengeti-Mara, } \\
\text { Tanzania \& Kenya) }{ }^{*}\end{array}$ & $\begin{array}{l}\text { 400-600 (Williamson et } \\
\text { al. 1988), 480 (1960s) } \\
\text { calculated from Sinclair } \\
\text { \& Norton-Griffiths } \\
\text { (1979) (Serengeti, } \\
\text { Tanzania)* }^{*}\end{array}$ & $\begin{array}{l}1.3 \text { million (Sinclair \& } \\
\text { Arcese 1995) }{ }^{*}, \\
8000-16000 \text { (Verlin- } \\
\text { den 1998), 25000 } \\
\text { (Serneels \& Lambin } \\
\text { 2001, Homewood et al. } \\
\text { 2001, Ottichilo et al. } \\
\text { 2001), } 25000(3)^{*}\end{array}$ & $\begin{array}{l}\sim 60000 \text { (Botswana - inferred from } \\
\text { Verlinden 1998); } 10000 \text { (between } \\
\text { 1970-1972; Kahurananga 1981), } \\
\text { 3000-7000 (1960-1970s; Sinclair \& } \\
\text { Norton-Griffiths 1979) (Serengeti, } \\
\text { Tanzania) }{ }^{*}, 6000 \text { (Whyte \& Joubert } \\
\text { 1988) (Kruger) }\end{array}$ & Thirgood et al. (2004) \\
\hline 0 (Estes 1991)* $^{*}$ & ? & 0 (Estes 1991)* $^{*}$ & $\begin{array}{l}\text { 1000s - South Africa's Karoo (Skinner } \\
\text { \& Smithers 1990, Skinner 1993), } \\
\text { 1000s (Estes 1991)* }\end{array}$ & Estes $(1991)^{*}$ \\
\hline$?$ & ? & 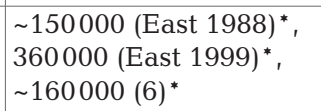 & ? & $\begin{array}{l}\text { Skinner \& Smithers } \\
(1990)^{*}, \text { Ottichilo et } \\
\text { al. }(2000)\end{array}$ \\
\hline $\begin{array}{l}200 \text { inferred (Sinclair \& } \\
\text { Norton-Griffiths } \\
1979)^{*}, 110 \text { (Kahu- } \\
\text { rananga \& Silk- } \\
\text { iluwasha 1997), } 400 \\
(3)^{*}\end{array}$ & $\begin{array}{l}\text { 240 calculated from } \\
\text { Sinclair \& Norton- } \\
\text { Griffiths (1979) } \\
\text { (Serengeti, Tanzania) }^{*}, \\
\text { 200-320 Namibia } \\
\text { (Ebedes 1972) 400+ }_{\text {(Grzimek \& Grzimek }} \\
\text { 1960), (Serengeti) }\end{array}$ & $\begin{array}{l}200000 \text { (Sinclair \& } \\
\text { Arcese 1995)* }, 3000 \\
(3)^{*}, \sim 10000(18) \\
\text { Tarangire ) }^{*}\end{array}$ & $\begin{array}{l}6000 \text { (between 1970-1972 - Kahu- } \\
\text { rananga 1981), } 23000 \text { (in 1955) } \\
\text { (Gasaway 1996) (Etosha), 200000 } \\
\text { 1960-1970 (Sinclair \& Norton- } \\
\text { Griffiths 1979) }\end{array}$ & Estes $(1991)^{*}$ \\
\hline 0 (Hack et al. 2002) $^{*}$ & ? & 0 (Hack et al. 2002) $^{*}$ & $\begin{array}{l}\text { 1000s - South Africa's Karoo (Skinner } \\
\text { 1993) }\end{array}$ & $\begin{array}{l}\text { Extinct (Hack et al. } \\
2002)^{*}\end{array}$ \\
\hline $\begin{array}{l}\sim 200 \text { (Campbell \& } \\
\text { Borner 1995*, Fryxell } \\
\text { et al. 2004, 2005) }\end{array}$ & $\begin{array}{l}\text { 160 calculated from } \\
\text { Sinclair \& Norton- } \\
\text { Griffiths (1979) } \\
\text { (Serengeti, Tanzania)* }^{*}\end{array}$ & 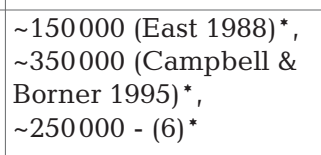 & $\begin{array}{l}725000 \text { (Bradley 1977, cited in } \\
\text { Sinclair \& Norton-Griffiths 1979) } \\
\text { (Serengeti, Tanzania) }^{*}\end{array}$ & $\begin{array}{l}\text { Sinclair \& Norton- } \\
\text { Griffiths (1979) } \\
\text { (Serengeti, Tanza- } \\
\text { nia) }{ }^{*}\end{array}$ \\
\hline $\begin{array}{l}\text { 300-400 (Fryxell \& } \\
\text { Sinclair 1988b) }\end{array}$ & $\begin{array}{l}\text { 300-400 (Fryxell \& } \\
\text { Sinclair 1988b) }\end{array}$ & $\begin{array}{l}\sim 1000000 \text { (East } \\
1988)^{*}, \sim 800000(6)^{*}\end{array}$ & >800000 (Fryxell \& Sinclair 1988b) & Estes $(1991)^{*}$ \\
\hline$?$ & ? & $\begin{array}{l}188 \text { (Campbell \& } \\
\text { Borner 1995) }\end{array}$ & 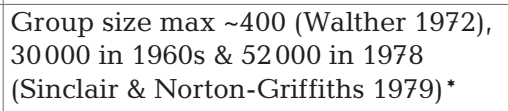 & Estes $(1991)^{*}$ \\
\hline
\end{tabular}


Table 1 (continued)

\begin{tabular}{|c|c|c|c|c|}
\hline \multirow{2}{*}{$\begin{array}{l}\text { Scientific } \\
\text { name }\end{array}$} & \multirow{2}{*}{$\begin{array}{l}\text { Common } \\
\text { name }\end{array}$} & \multirow[t]{2}{*}{ Location } & \multicolumn{2}{|l|}{ Mass migration } \\
\hline & & & Current & Historic \\
\hline $\begin{array}{l}\text { Oryx } \\
\text { dammah }\end{array}$ & $\begin{array}{l}\text { Scimitar } \\
\text { horned } \\
\text { oryx }\end{array}$ & Africa & & $\begin{array}{l}\text { 36 - Mallon \& Kingswood (2001) } \\
\text { (Chad \& Niger)* }^{*}, \mathbf{3 7} \text { - CMS } \\
\text { (2006b) (Mauritania-Morocco, } \\
\text { Mali-Niger, Niger-Algeria, Mali- } \\
\text { Burkina Faso, Niger-Chad, } \\
\text { Chad-Sudan)** }^{*}\end{array}$ \\
\hline $\begin{array}{l}\text { Tragela- } \\
\text { phus oryx }\end{array}$ & Eland & Africa & $\begin{array}{l}33 \text { - Campbell \& Borner (1995) (Serengeti-Mara, Tanzania \& } \\
\text { Kenya) }^{*}, \mathbf{3} \text { - Verlinden (1998) (Kalahari, Botswana) \& (1) } \\
\text { (Kalahari, Botswana) }^{*}\end{array}$ & $?$ \\
\hline $\begin{array}{l}\text { Antilo- } \\
\text { carpa } \\
\text { americana }\end{array}$ & $\begin{array}{l}\text { Prong- } \\
\text { horn }\end{array}$ & $\begin{array}{l}\text { North } \\
\text { America }\end{array}$ & 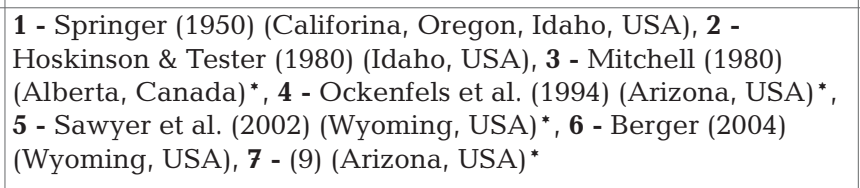 & Berger (2004) \\
\hline $\begin{array}{l}\text { Bison } \\
\text { bison }\end{array}$ & Bison & $\begin{array}{l}\text { North } \\
\text { America }\end{array}$ & $\begin{array}{l}\text { Van Vuren \& Bray (1986), } 8 \text { - Gates et al. (2001) (Alberta, } \\
\text { Canada) }{ }^{*}, \mathbf{9} \text { - (10) (Yellowstone, USA)* }\end{array}$ & Dary (1974) (USA)* \\
\hline $\begin{array}{l}\text { Cervus } \\
\text { elaphus }\end{array}$ & Elk & $\begin{array}{l}\text { North } \\
\text { America }\end{array}$ & 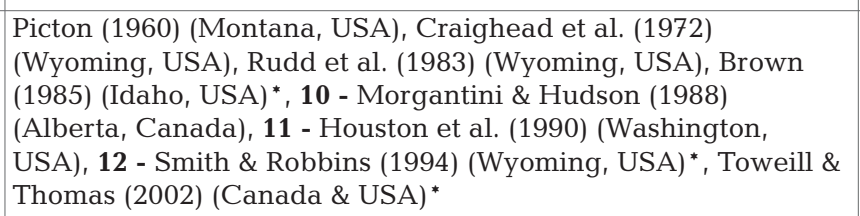 & $\begin{array}{l}\text { Skinner (1925) (Wyoming, USA), } \\
\text { Craighead et al. (1972), Berger } \\
\text { (2004) (Wyoming, USA) }\end{array}$ \\
\hline $\begin{array}{l}\text { Rangifer } \\
\text { tarandus }\end{array}$ & $\begin{array}{l}\text { Caribou/ } \\
\text { reindeer }\end{array}$ & $\begin{array}{l}\text { North } \\
\text { America \& } \\
\text { Eurasia }\end{array}$ & 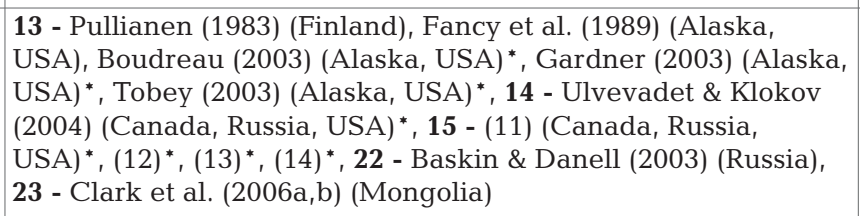 & $\begin{array}{l}\text { Ulvevadet \& Klokov (2004) } \\
\text { Baskin \& Danell (2003) (Russia) }\end{array}$ \\
\hline $\begin{array}{l}\text { Capreolus } \\
\text { pygargus }\end{array}$ & $\begin{array}{l}\text { Siberian } \\
\text { roe deer }\end{array}$ & Eurasia & 16 - Danilkin (1996) (China, Russia) ${ }^{*}$ & $\begin{array}{l}17 \text { - Kirikov (1959) (Ukraine) }{ }^{*}, \\
\text { Danilkin }(1996)^{*}, \mathbf{2 4} \text { - Baskin } \& \\
\text { Danell }(2003)(\text { Ural })^{*}\end{array}$ \\
\hline $\begin{array}{l}\text { Pantholops } \\
\text { hodgsoni }\end{array}$ & Chiru & Eurasia & $\begin{array}{l}18 \text { - Schaller (1998) (Chang Tang \& Qinghai, China) }{ }^{*} \text {, Lian et } \\
\text { al. (2005) (Kekexili, China) }\end{array}$ & Schaller (1998)* \\
\hline $\begin{array}{l}\text { Equus } \\
\text { hemionus }\end{array}$ & $\begin{array}{l}\text { Kulan/ } \\
\text { khulan }\end{array}$ & Eurasia & ? & $\begin{array}{l}25 \text { - Baskin \& Danell (2003) } \\
\text { (Kazakhstan) }^{*}\end{array}$ \\
\hline $\begin{array}{l}\text { Procapra } \\
\text { gutturosa }\end{array}$ & $\begin{array}{l}\text { Mongo- } \\
\text { lian } \\
\text { gazelle }\end{array}$ & Eurasia & $\begin{array}{l}\text { Lhagvasuren \& Milner-Gulland (1997) (Mongolia-China), Jiang } \\
\text { et al. (1998) (Mongolia-China/Russia), Baskin \& Danell (2003) } \\
\text { (Mongolia-Russia)*, } 26 \text { - Ito et al. (2005) (Dornogobi, Mongolia), } \\
\mathbf{1 9} \text { - Ito et al. (2006) (Dornogobi \& Omnogobi, Mongolia), 27 - } \\
\text { Olson et al. (2005), } 28 \text { - Mueller et al. (2008) (Eastern Mongolia) }\end{array}$ & Jiang et al. (1998) \\
\hline $\begin{array}{l}\text { Saiga } \\
\text { tatarica } \\
\text { tatarica \& } \\
\text { S. } t \text {. } \\
\text { mongolica }\end{array}$ & Saiga & Eurasia & $\begin{array}{l}\text { Eregdendagvaa (1954), } 20 \text { - Bekenov et al. (1998) (Kazakhstan), } \\
\mathbf{2 1} \text { - Lushchekina et al. (1999) (Mongolia), Milner-Gulland et al. } \\
(2001) \text { (Kazakhstan, Mongolia), } 29 \text { - Baskin \& Danell (2003) } \\
\text { (Kalmykia, Russia)*, 30 - CMS (2006a) (Russia)*, } 31 \text { - Berger et } \\
\text { al. (2008a), } 32 \text { - Berger et al. (2008b) (Mongolia) }\end{array}$ & $\begin{array}{l}\text { Eregdendagvaa (1954)* } \\
\text { Bekenov et al. (1998), Baskin \& } \\
\text { Danell }(2003)^{*}\end{array}$ \\
\hline
\end{tabular}

(1) C. Thouless (unpubl.) \& R. Brett (pers. comm.)

(2) J. O. Ogutu (pers. comm.)

(3) www.african-parks.org/apffoundation/index.php?option=com_content\&task=view\&id=48\&Itemid=83

(4) M. van Vewalle (unpubl. thesis) \& N. Owen-Smith (pers. comm.)

(5) J. O. Ogutu \& M. Norton-Griffiths (pers. comm.)

(6) www.msnbc.msn.com/id/19191023/

(7) S. Mohsarif (pers. comm.)

(8) www.ultimateungulate.com/Artiodactyla/oryx_dammah.html

(9) R. A. Ockenfels (pers. comm.) 


\begin{tabular}{|c|c|c|c|c|}
\hline \multirow{2}{*}{$\begin{array}{l}\text { Current migration distance } \\
\text { (round trip, } \mathrm{km} \text { ) }\end{array}$} & \multirow{2}{*}{$\begin{array}{l}\text { Historic migration dis- } \\
\text { tance (round trip, km) }\end{array}$} & \multicolumn{2}{|l|}{ Numbers migrating } & \multirow{2}{*}{$\begin{array}{l}\text { Non-migratory } \\
\text { reference }\end{array}$} \\
\hline & & Current & Historic & \\
\hline $\begin{array}{l}0 \text { (Mallon \& Kingswood } \\
2001)^{*}\end{array}$ & 100s (CMS 2006b) & 0 (Mallon \& Kingswood 2001) $^{*}$ & 1000 's - (8) & $\begin{array}{l}\text { Extinct in wild } \\
\text { (Mallon \& Kingswood } \\
2001)^{*}\end{array}$ \\
\hline $\begin{array}{l}400 \text { (Campbell \& Borner }^{*} \\
1995)^{*}\end{array}$ & $?$ & $\begin{array}{l}12000 \text { in groups of } 200 \text { (Campbell \& } \\
\text { Borner 1995), (16) } 16000(1)^{*}\end{array}$ & $?$ & $?$ \\
\hline 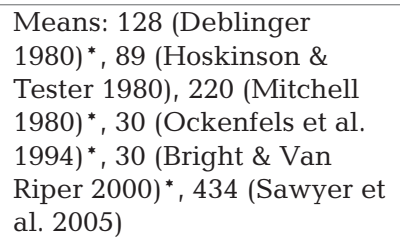 & $?$ & $\begin{array}{l}\sim 40 \text { (Mitchell 1980) }^{*}, 2000 \text { (Sawyer et } \\
\text { al. 2002) }\end{array}$ & $?$ & $\begin{array}{l}\text { Hoskinson \& Tester } \\
(1980), \text { Sawyer et al. } \\
(2002)^{*}\end{array}$ \\
\hline $\begin{array}{l}50 \text { (Van Vuren \& Bray 1986), } \\
160 \text { (Gates et al. 2001) }^{*} \\
\operatorname{max.} 80(10)^{*}\end{array}$ & $?$ & $\begin{array}{l}\sim 600 \text { (McDonald 1981)* }{ }^{*} \sim 300 \text { (Van } \\
\text { Vuren \& Bray 1986), } 1900 \text { (Larter et } \\
\text { al. 2000), 2151 (Joly \& Messier 2001) }\end{array}$ & $?$ & Dary $(1974)^{*}$ \\
\hline $\begin{array}{l}70 \text { (Craighead et al. 1972), } \\
73 \text { (Morgantini \& Hudson } \\
\text { 1988), } 60 \text { (Houston et al. } \\
\text { 1990), } 200 \text { (Smith \& Robbins } \\
\text { 1994)* }\end{array}$ & $?$ & $\begin{array}{l}\text { 250-1000 (Craighead et al. 1972), } 1000 \\
\text { (Morgantini \& Hudson 1988), 400-1000 } \\
\text { (Houston et al. 1990), 11000 (Smith \& } \\
\text { Robbins 1994)* }\end{array}$ & $?$ & $\begin{array}{l}\text { Toweill \& Thomas } \\
(2002)^{*}\end{array}$ \\
\hline $\begin{array}{l}3031 \text { (mean) (Fancy et al. } \\
\text { 1989), 1000-1200 Taymyr } \\
\text { (Russia), 600-700 Yakutia } \\
\text { (Russia), 80-150, Altai } \\
\text { (Russia) (Baskin \& Danell } \\
\text { 2003) }\end{array}$ & $?$ & $\begin{array}{l}\sim 200000 \text { (Boudreau 2003) }^{*}, \sim 40000 \\
\text { (Gardner 2003) }^{*}, \sim 3000{\text { (Tobey } 2003)^{*}}^{*} \\
\text { maximum observed group size during } \\
\text { migration } 20000 \text { (Baskin \& Danell } \\
\text { 2003) (Taymyr, Russia) }\end{array}$ & $?$ & $\begin{array}{l}\text { Ulvevadet \& Klokov } \\
(2004)^{*}, \text { Baskin \& } \\
\text { Danell }(2003)\end{array}$ \\
\hline $\begin{array}{l}\text { 200-400 (Danilkin et al. } \\
\text { 1992), Most 200, max. } \\
800-1000 \text { (Danilkin 1996) }^{*}\end{array}$ & $?$ & $\begin{array}{l}\text { 25000-30 } 000 \text { (Danilkin et al. 1992) } \\
10000 \text { with bunches up to } 200-1000 \\
{\text { (Danilkin } 1996)^{*}}^{*}\end{array}$ & $?$ & Danilkin $(1996)^{*}$ \\
\hline 500-600 (Schaller 1998)* & $?$ & $\begin{array}{l}\sim 2000, \text { Some few 100s to } 700 \text { (Schaller } \\
\text { 1998)* } \sim 3000 \text { (Lian et al. 2005, Lian et } \\
\text { al. 2007) (Kekexili only), } \sim 100000(17)^{*}\end{array}$ & $?$ & Schaller $(1998)^{*}$ \\
\hline$?$ & $?$ & $?$ & $\begin{array}{l}\text { Groups up to } \\
\sim 200 \text { (Baskin \& } \\
\text { Danell (2003)) }\end{array}$ & $\begin{array}{l}\text { Reading et al. (2001), } \\
\text { Kaczensky et al. } \\
(2006)^{*}\end{array}$ \\
\hline $\begin{array}{l}\text { 100-1000s (Ito et al. 2005, } \\
2006)\end{array}$ & $?$ & $\begin{array}{l}800000-900000 \text { (Olson et al. 2005), } \\
\text { maximum herd size } 80000 \text { (Lhagva- } \\
\text { suren \& Milner-Gulland 1997) }\end{array}$ & $?$ & $\begin{array}{l}\text { No large-scale } \\
\text { migrations in } \\
\text { Mongolia (Clark et } \\
\text { al. 2006b*, Mueller et } \\
\text { al. 2008) }\end{array}$ \\
\hline $\begin{array}{l}150 \text { (Eregdendagvaa 1954) }^{*}, \\
400-2400 \text { (Bekenov et al. } \\
\text { 1998), 300-800 in Kalmykia, } \\
\text { 250-300 Volga-Ural, } \\
\text { 300-400 Ustyurt, 500-600 } \\
\text { Betpagdala-Turgay (Baskin } \\
\text { \& Danell 2003)* }\end{array}$ & $?$ & $\begin{array}{l}1500 \text { (Clark et al. 2006a, Wingard \& } \\
\text { Zahler 2006) }{ }^{*},<70000 \text { (total) (CMS } \\
\text { 2006a) }^{*}\end{array}$ & $?$ & $?$ \\
\hline
\end{tabular}

(10) P. Gogan (pers. comm.)

(11) www.carmanetnetwork.com/display/public/home

(12) www.arctic-caribou.com/

(13) www.wkss.nt.ca/HTML/08_ProjectsReports/PDF/SeasonalMovementsFinal.pdf

(14) http://arctic.fws.gov/cariboumaps.htm

(15) http://library.thinkquest.org/16645/wildlife/grants_gazelle.shtml?tqskip1=1

(16) M. Borner (pers. comm.)

(17) http://news.nationalgeographic.com/news/2007/02/070206-tibet-antelope.html

(18) Tanzania Wildlife Research Institute (unpubl.) 
Table 2. Summary of ecological drivers, threats, and state of knowledge necessary to conserve aggregated migrations for terrestrial mammals. Grasslands quality/quantity refers to forage 'green-up' as a result of rainfall or fire; vegetation green-up refers to a green-up spanning elevation gradients. \#1 to 6 in 'State of conservation knowledge' refer to Steps 1 to 6 in the framework for conservation. Num-

\begin{tabular}{|c|c|c|c|c|c|c|c|}
\hline \multirow[t]{2}{*}{ Scientific name } & \multirow{2}{*}{$\begin{array}{l}\text { Common } \\
\text { name }\end{array}$} & \multirow[t]{2}{*}{ Location } & \multicolumn{5}{|c|}{ Ecological drivers } \\
\hline & & & $\begin{array}{l}\text { Grasslands quality/quan- } \\
\text { tity }\end{array}$ & Surface water & Snow depth & $\begin{array}{l}\text { Vegetation } \\
\text { green-up }\end{array}$ & Traditional areas \\
\hline $\begin{array}{l}\text { Alcelaphus } \\
\text { buselaphus }\end{array}$ & Hartebeest & Africa & $\begin{array}{l}\text { Skinner \& Smithers } \\
(1990)^{*}, \text { Estes }(1991)^{*}, \\
\text { Verlinden }(1998){ }^{\prime}\end{array}$ & $\begin{array}{l}\text { Skinner \& } \\
\text { Smithers }(1990)^{*}\end{array}$ & & & \\
\hline $\begin{array}{l}\text { Antidorcas } \\
\text { marsupialis }\end{array}$ & Springbok $^{\mathrm{b}}$ & Africa & $\begin{array}{l}\text { Skinner \& Smithers } \\
(1990)^{*}, \text { Skinner }(1993), \\
\text { Skinner \& Louw }(1996)^{*} \text {, } \\
\text { Verlinden (1998) }\end{array}$ & & & & \\
\hline $\begin{array}{l}\text { Connochaetes } \\
\text { gnou }\end{array}$ & $\begin{array}{l}\text { Black } \\
\text { wildebeest }\end{array}$ & Africa & $\begin{array}{l}\text { Estes }(1991)^{*}, \text { Skinner } \\
(1993)\end{array}$ & & & & \\
\hline $\begin{array}{l}\text { Connochaetes } \\
\text { taurinus }\end{array}$ & $\begin{array}{l}\text { Blue } \\
\text { wildebeest }\end{array}$ & Africa & $\begin{array}{l}\text { Talbot \& Talbot (1963), } \\
\text { Skinner \& Smithers } \\
(1990)^{*}, \text { Kahurananga \& } \\
\text { Silkiluwasha (1997), } \\
\text { Mduma et al. (1999), } \\
\text { Boone et al. (2006) }\end{array}$ & $\begin{array}{l}\text { Williamson et al. } \\
\text { (1988), Verlinden } \\
(1998)\end{array}$ & & & \\
\hline $\begin{array}{l}\text { Damaliscus } \\
\text { dorcas }\end{array}$ & Blesbok & Africa & 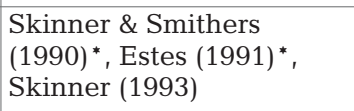 & & & & \\
\hline $\begin{array}{l}\text { Damaliscus } \\
\text { lunatus }\end{array}$ & Tiang (topi) & Africa & & & & & \\
\hline $\begin{array}{l}\text { Equus } \\
\text { burchellii }\end{array}$ & $\begin{array}{l}\text { Burchell's } \\
\text { zebra }\end{array}$ & Africa & $\begin{array}{l}\text { Smuts }(1972)^{*}, \text { Skinner \& } \\
\text { Smithers }(1990)^{*}, \text { Sinclair } \\
\text { \& Norton-Griffiths } \\
(1979)^{*}, \text { Gasaway et al. } \\
(1996), \text { Kahurananga \& } \\
\text { Silkiluwasha (1997) }\end{array}$ & $\begin{array}{l}\text { Smuts }(1972)^{*}, \\
\text { Skinner \& } \\
\text { Smithers (1990)*, } \\
\text { Kahurananga \& } \\
\text { Silkiluwasha } \\
\text { (1997) }\end{array}$ & & & \\
\hline $\begin{array}{l}\text { Equus quagga } \\
\text { quagga }\end{array}$ & Quagga & Africa & $\begin{array}{l}\text { Estes }(1991)^{*}, \text { Skinner } \\
(1993)\end{array}$ & & & & \\
\hline $\begin{array}{l}\text { Gazella } \\
\text { thomsonii \& G. } \\
\text { t. albonotata }\end{array}$ & $\begin{array}{l}\text { Thompson's } \\
\text { gazelle \& } \\
\text { Mongalla } \\
\text { gazelle }\end{array}$ & Africa & $\begin{array}{l}\text { Sinclair \& Norton- } \\
\text { Griffiths }(1979)^{*}\end{array}$ & & & & \\
\hline Kobus kob & $\begin{array}{l}\text { White- } \\
\text { eared kob }\end{array}$ & Africa & Fryxell \& Sinclair (1988b) & $\begin{array}{l}\text { Fryxell \& Sinclair } \\
(1988 b)\end{array}$ & & & \\
\hline Nanger granti & $\begin{array}{l}\text { Grant's } \\
\text { gazelle }\end{array}$ & Africa & Walther (1972), (15)* & & & & \\
\hline Oryx dammah & $\begin{array}{l}\text { Scimitar } \\
\text { horned oryx }\end{array}$ & Africa & & & & & \\
\hline
\end{tabular}


bers 1 to 18 in parentheses indicate an unpublished source, listed as a footnote to Table 1. An asterisk indicates literature which may not have undergone peer review

\begin{tabular}{|c|c|c|c|c|c|c|}
\hline \multicolumn{6}{|c|}{ Threat } & \multirow{2}{*}{$\begin{array}{l}\text { State of conservation } \\
\text { knowledge }\end{array}$} \\
\hline Fencing & $\begin{array}{l}\text { Livestock } \\
\text { encroachment }\end{array}$ & $\begin{array}{l}\text { Human encroach- } \\
\text { ment (including } \\
\text { agricultural } \\
\text { expansion) }\end{array}$ & $\begin{array}{l}\text { Over hunting/ } \\
\text { poaching }\end{array}$ & $\begin{array}{l}\text { Energy } \\
\text { develop- } \\
\text { ment }\end{array}$ & $\begin{array}{l}\text { Trans- } \\
\text { portation } \\
\text { corridors }\end{array}$ & \\
\hline Verlinden (1998) & $\begin{array}{l}\text { Williamson et al. } \\
\text { (1988), Verlinden } \\
\text { (1998) }\end{array}$ & & & & & $\begin{array}{l}\text { Verlinden (1998) (Kalahari, } \\
\text { Botswana) - \#1, (1) (Kalahari, } \\
\text { Botswana)* - \#1 }\end{array}$ \\
\hline $\begin{array}{l}\text { Skinner \& Smithers } \\
(1990)^{*}, \text { Estes } \\
(1991)^{*}\end{array}$ & $\begin{array}{l}\text { Williamson et al. } \\
(1988), \text { Estes } \\
(1991)^{*} \text { Verlinden } \\
(1998)\end{array}$ & $\begin{array}{l}\text { Skinner \& } \\
\text { Smithers }(1990)^{*}, \\
\text { Estes }(1991)^{*}\end{array}$ & Skinner (1993) & & & 0 \\
\hline Estes $(1991)^{*}$ & Estes $(1991)^{*}$ & $\begin{array}{l}\text { Skinner \& } \\
\text { Smithers }(1990)^{*}, \\
\text { Estes }(1991)^{*}\end{array}$ & $\begin{array}{l}\text { Skinner \& } \\
\text { Smithers }(1990)^{*}\end{array}$ & & & 0 \\
\hline $\begin{array}{l}\text { Berry (1997), } \\
\text { Williamson et al. } \\
\text { (1988), Whyte \& } \\
\text { Joubert (1988), } \\
\text { Spinage (1992), } \\
\text { Gasaway et al. } \\
\text { (1996), Verlinden } \\
\text { (1998) }\end{array}$ & $\begin{array}{l}\text { Williamson et al. } \\
\text { (1988), Homewood } \\
\text { et al. (2001) }\end{array}$ & $\begin{array}{l}\text { Williamson et al. } \\
\text { (1988), Kahu- } \\
\text { rananga \& } \\
\text { Silkiluwasha } \\
\text { (1997), Home- } \\
\text { wood et al. (2001), } \\
\text { Thirgood et al. } \\
\text { (2004) }\end{array}$ & $\begin{array}{l}\text { Gasaway et al. } \\
\text { (1996), Thirgood } \\
\text { et al. (2004) }\end{array}$ & & & $\begin{array}{l}\text { Williamson et al. (1988) (Kala- } \\
\text { hari, Botswana) - \#4, Bonifica } \\
\text { (1993) (Botswana) }{ }^{*} \text { - \#1, Kahu- } \\
\text { rananga \& Silkiluwasha (1997) } \\
\text { (Tarangire National Park, } \\
\text { Tanzania) - \#4, Verlinden (1998) } \\
\text { (Kalahari, Botswana) - \#1, (1) } \\
\text { (Kalahari, Botswana)* - \#1, } \\
\text { Homewood et al. (2001) } \\
\text { (Serengeti, Tanzania) - \#4, } \\
\text { Serneels \& Lambin (2001) } \\
\text { (Serengeti-Mara, Tanzania \& } \\
\text { Kenya) - \#5, Thirgood et al. } \\
\text { (2004) (Serengeti, Tanzania) - \#4, } \\
\text { Boone et al. (2006) (Serengeti, } \\
\text { Tanzania) - \#2, Thirgood et al. } \\
\text { (2008) (Serengeti, Tanzania) - \#6 }\end{array}$ \\
\hline \multirow[t]{2}{*}{ Estes $(1991)^{*}$} & Estes $(1991)^{*}$ & Estes $(1991)^{*}$ & $\begin{array}{l}\text { Skinner \& } \\
\text { Smithers (1990)* }\end{array}$ & & & 0 \\
\hline & & & East (1999) ${ }^{*}$ & & & 0 \\
\hline $\begin{array}{l}\text { Berry }(1980)^{*}, \\
\text { Whyte \& Joubert } \\
(1988)\end{array}$ & $\begin{array}{l}\text { Whyte \& Joubert } \\
\text { (1988) }\end{array}$ & $\begin{array}{l}\text { Kahurananga \& } \\
\text { Silkiluwasha } \\
(1997)\end{array}$ & & & & $\begin{array}{l}\text { Sinclair \& Norton-Griffiths (1979) } \\
\text { (Serengeti, Tanzania) }^{*} \text { - \#1, } \\
\text { Kahurananga \& Silkiluwasha } \\
\text { (1997) (Tarangire National Park, } \\
\text { Tanzania) - \#4 }\end{array}$ \\
\hline \multirow[t]{5}{*}{ Estes $(1991)^{*}$} & $\begin{array}{l}\text { Estes }(1991)^{*} \\
\text { Hack et al. }(2002)^{*}\end{array}$ & Estes $(1991)^{*}$ & $\begin{array}{l}\text { Estes }(1991)^{*} \\
\text { Hack et al. }(2002)^{*}\end{array}$ & & & 0 \\
\hline & & & & & & $\begin{array}{l}\text { Campbell \& Borner (1995) } \\
\text { (Serengeti-Mara, Tanzania \& } \\
\text { Kenya) }^{*} \text { - \#1 }\end{array}$ \\
\hline & & & & & & 0 \\
\hline & & & & & & 0 \\
\hline & $\begin{array}{l}\text { Mallon \& } \\
\text { Kingswood } \\
(2001)^{*}\end{array}$ & & $\begin{array}{l}\text { Mallon \& } \\
\text { Kingswood } \\
(2001)^{*}\end{array}$ & & & 0 \\
\hline
\end{tabular}


Table 2 (continued)

\begin{tabular}{|c|c|c|c|c|c|c|c|}
\hline \multirow[t]{2}{*}{ Scientific name } & \multirow{2}{*}{$\begin{array}{l}\text { Common } \\
\text { name }\end{array}$} & \multirow[t]{2}{*}{ Location } & \multicolumn{5}{|c|}{ Ecological drivers } \\
\hline & & & $\begin{array}{l}\text { Grasslands } \\
\text { quality/quan- } \\
\text { tity }\end{array}$ & Surface water & Snow depth & $\begin{array}{l}\text { Vegetation green- } \\
\text { up }\end{array}$ & Traditional areas \\
\hline $\begin{array}{l}\text { Tragelaphus } \\
\text { oryx }\end{array}$ & Eland & Africa & & & & & \\
\hline $\begin{array}{l}\text { Antilocarpa } \\
\text { americana }\end{array}$ & Pronghorn ${ }^{\mathrm{a}, \mathrm{c}}$ & $\begin{array}{l}\text { North } \\
\text { America }\end{array}$ & $\begin{array}{l}\text { Yoakum } \\
(1978)^{*}, \\
\text { Hoskinson \& } \text { Tester (1980), } \\
\text { Ockenfels et } \\
\text { al. }(1994)^{*}\end{array}$ & Yoakum (1978)* & $\begin{array}{l}\text { Hoskinson \& } \\
\text { Tester (1980) }\end{array}$ & & \\
\hline Bison bison & Bison & $\begin{array}{l}\text { North } \\
\text { America }\end{array}$ & & & $\begin{array}{l}\text { Meagher (1989), } \\
\text { Frank \& } \\
\text { McNaughton } \\
(1992)\end{array}$ & $\begin{array}{l}\text { Frank \& } \\
\text { McNaughton } \\
(1992)\end{array}$ & \\
\hline Cervus elaphus & Elk & $\begin{array}{l}\text { North } \\
\text { America }\end{array}$ & & & $\begin{array}{l}\text { Rudd et al. (1983), } \\
\text { Morgantini \& } \\
\text { Hudson (1988) }\end{array}$ & $\begin{array}{l}\text { Houston et al. } \\
\text { (1990), Morgantini } \\
\text { \& Hudson (1988), } \\
\text { Frank (1998) }\end{array}$ & $\begin{array}{l}\text { Morgantini \& } \\
\text { Hudson (1988) }\end{array}$ \\
\hline $\begin{array}{l}\text { Rangifer } \\
\text { tarandus }\end{array}$ & $\begin{array}{l}\text { Caribou/ } \\
\text { reindeer }\end{array}$ & $\begin{array}{l}\text { North } \\
\text { America } \\
\text { \& Eurasia }\end{array}$ & & & $\begin{array}{l}\text { Ulvevadet \& } \\
\text { Klokov }(2004)^{*}, \\
(11)^{*}\end{array}$ & $\begin{array}{l}\text { Ulvevadet \& } \\
\text { Klokov }(2004)^{*}, \\
(11)^{*}\end{array}$ & $\begin{array}{l}\text { Ulvevadet \& } \\
\text { Klokov }(2004)^{*}, \\
(11)^{*}\end{array}$ \\
\hline $\begin{array}{l}\text { Capreolus } \\
\text { pygargus }\end{array}$ & $\begin{array}{l}\text { Siberian roe } \\
\text { deer }\end{array}$ & Eurasia & & & $\begin{array}{l}\text { Danilkin et al. } \\
(1992), \text { Danilkin } \\
(1996)^{*}\end{array}$ & & $\begin{array}{l}\text { Danilkin et al. } \\
(1992), \text { Danilkin } \\
(1996)^{*}\end{array}$ \\
\hline $\begin{array}{l}\text { Pantholops } \\
\text { hodgsoni }\end{array}$ & Chiru & Eurasia & & & Schaller (1998)* & & Schaller (1998)* \\
\hline $\begin{array}{l}\text { Equus } \\
\text { hemionus }\end{array}$ & $\begin{array}{l}\text { Kulan/ } \\
\text { khulan }\end{array}$ & Eurasia & $\begin{array}{l}\text { Kaczensky et } \\
\text { al. }(2006)^{*}\end{array}$ & $\begin{array}{l}\text { Baskin \& Danell } \\
(2003)^{*}, \\
\text { Kaczensky et al. } \\
(2006)^{*}\end{array}$ & 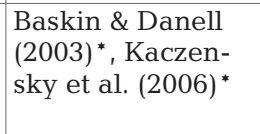 & & \\
\hline $\begin{array}{l}\text { Procapra } \\
\text { gutturosa }\end{array}$ & $\begin{array}{l}\text { Mongolian } \\
\text { gazelle }\end{array}$ & Eurasia & $\begin{array}{l}\text { Leimgruber } \\
\text { et al. (2001), } \\
\text { Mueller et al. } \\
(2008)\end{array}$ & & Jiang et al. (1998) & & \\
\hline $\begin{array}{l}\text { Saiga tatarica } \\
\text { tatarica \& S. } t . \\
\text { mongolica }\end{array}$ & Saiga & Eurasia & $\begin{array}{l}\text { Bekenov et } \\
\text { al. (1998), } \\
\text { Baskin \& } \\
\text { Danell } \\
(2003)^{*}\end{array}$ & $\begin{array}{l}\text { Bekenov et al. } \\
(1998)\end{array}$ & $\begin{array}{l}\text { Bekenov et al. } \\
(1998)\end{array}$ & & \\
\hline
\end{tabular}

${ }^{a}$ Uncharted ecological driver is forage moisture (Hoskinson \& Tester 1980)

${ }^{b}$ Uncharted threat is rinderpest (Skinner 1993)
'Uncharted threat is brush and tree invasion (Ockenfels et al. 1994*) dUncharted threats are climate change \& disturbing of calving areas (Ulvevadet \& Klokov 2004* , www.carmanetwork.com/display/public/home) 


\begin{tabular}{|c|c|c|c|c|c|c|}
\hline \multicolumn{6}{|c|}{ Threat } & \multirow{2}{*}{$\begin{array}{l}\text { State of conservation } \\
\text { knowledge }^{\mathrm{c}}\end{array}$} \\
\hline Fencing & $\begin{array}{l}\text { Livestock } \\
\text { encroachment }\end{array}$ & $\begin{array}{l}\text { Human encroachment } \\
\text { (including agricultural } \\
\text { expansion) }\end{array}$ & $\begin{array}{l}\text { Over hunting / } \\
\text { poaching }\end{array}$ & $\begin{array}{l}\text { Energy } \\
\text { development }\end{array}$ & \begin{tabular}{|l|} 
Trans- \\
portation \\
corridors
\end{tabular} & \\
\hline & & $\begin{array}{l}\text { Campbell \& Borner } \\
(1995)^{*}\end{array}$ & $\begin{array}{l}\text { Campbell \& Borner } \\
(1995)^{*}\end{array}$ & & & $\begin{array}{l}\text { Campbell \& Borner (1995) } \\
\text { (Serengeti-Mara, Tanzania \& } \\
\text { Kenya) }^{*} \text { - \#1, (1) (Kalahari, } \\
\text { Botswana) }^{*} \text { - \#1 }\end{array}$ \\
\hline $\begin{array}{l}\text { Ockenfels et } \\
\text { al. }(1994)^{*}, \\
\text { Berger }(2004)\end{array}$ & & $\begin{array}{l}\text { Hoskinson \& Tester } \\
(1980), \text { Ockenfels et } \\
\text { al. }(1994)^{*}, \text { Sawyer et } \\
\text { al. }(2002)^{*}, \text { Berger } \\
(2004)\end{array}$ & & 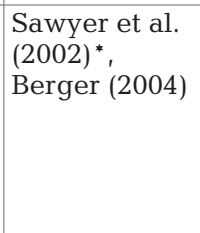 & $\begin{array}{l}\text { Ocken- } \\
\text { fels et al. } \\
(1994)^{*}\end{array}$ & $\begin{array}{l}\text { Ockenfels et al. (1994) (Arizona, } \\
\text { USA)* - \#4, Sawyer et al. }^{*} \text { (2002) (Wyoming, USA)* - \#1, }_{\text {Berger (2004) (Wyoming, USA) }} \\
\text { - \#5, Berger et al. (2006) } \\
\text { (Wyoming, USA) - \#5, (9) } \\
\text { (Arizona, USA)* - \#1 }\end{array}$ \\
\hline Berger (2001) & & & $\begin{array}{l}\text { Dary }(1974)^{*} \\
\text { Berger }(2001)\end{array}$ & & & $\begin{array}{l}\text { Gates et al. (2001) (Alberta, } \\
\text { Canada)* - \#4, (10) (Yellow- } \\
\text { stone, USA)* - \#5 }\end{array}$ \\
\hline \multirow[t]{4}{*}{$\begin{array}{l}\text { Toweill \& } \\
\text { Thomas } \\
(2002)^{*}, \\
\text { Berger }(2004)\end{array}$} & & $\begin{array}{l}\text { Houston et al. (1990), } \\
\text { Toweill \& Thomas } \\
(2002)^{*}, \text { Berger }(2004)\end{array}$ & $\begin{array}{l}\text { Houston et al. } \\
\text { (1990), Morgantini } \\
\text { \& Hudson (1988) }\end{array}$ & Berger (2004) & & 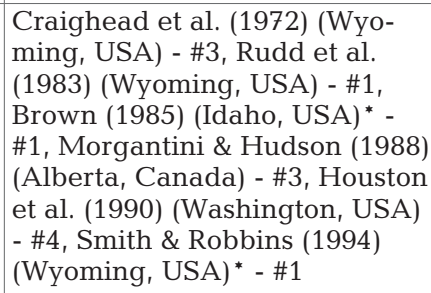 \\
\hline & & & $\begin{array}{l}\text { Ulvevadet \& Klokov } \\
(2004)^{*},(11)^{*}\end{array}$ & $\begin{array}{l}\text { Ulvevadet \& } \\
\text { Klokov } \\
(2004)^{*},(11)^{*}\end{array}$ & & $\begin{array}{l}\text { Ulvevadet \& Klokov (2004) } \\
\text { (Canada, Russia, USA)* - \#4, }(11)(\text { Canada, Russia, USA) } \\
\text { - \#4, Baskin \& Danell }(2003)^{*} \text { - \#1 }\end{array}$ \\
\hline & & Danilkin (1996) $^{*}$ & $\begin{array}{l}\text { Kirikov (1959)*, } \\
\text { Danilkin (1996)*, } \\
\text { Baskin \& Danell } \\
(2003)^{*}\end{array}$ & & & $\begin{array}{l}\text { Danilkin (1996) (China, Russia) }{ }^{*} \\
\text { - \#4 }\end{array}$ \\
\hline & & & $\begin{array}{l}\text { Schaller (1998)* } \\
\text { Lian et al. (2005) }\end{array}$ & & & $\begin{array}{l}\text { Lian et al. (2005) (Kekexili, } \\
\text { China) - \#2 }\end{array}$ \\
\hline $\begin{array}{l}\text { Kaczensky et } \\
\text { al. }(2006)^{*}\end{array}$ & $\begin{array}{l}\text { Reading et al. } \\
(2001), \\
\text { Kaczensky et } \\
\text { al. }(2006)^{*}\end{array}$ & Clark et al. $(2006 b)^{*}$ & $\begin{array}{l}\text { Reading et al } \\
(2001), \text { Kaczensky } \\
\text { et al. }(2006)^{*}, \text { Clark } \\
\text { et al. }(2006))^{*}\end{array}$ & & $\begin{array}{l}\text { Kaczen- } \\
\text { sky et al. } \\
(2006)^{*}\end{array}$ & Kaczensky et al. (2006) ${ }^{*}-\# 4$ \\
\hline $\begin{array}{l}\text { Ito et al (2005), } \\
\text { Clark et al. } \\
(2006 b)^{*}\end{array}$ & $\begin{array}{l}\text { Yoshihara et } \\
\text { al. (2008) }\end{array}$ & & $\begin{array}{l}\text { Reading et al. } \\
(1998), \text { Clark et al. } \\
(2006 b)^{*}, \text { Wingard } \\
\& \text { Zahler }(2006)^{*}\end{array}$ & & $\begin{array}{l}\text { Ito et al. } \\
(2005) \\
(2008)\end{array}$ & $\begin{array}{l}\text { Lhagvasuren \& Milner-Gulland } \\
\text { (1997) - \#2, Jiang et al. (1998) - } \\
\text { \#1, Ito et al. (2006) (Dornogobi } \\
\text { \& Omnogobi, Mongolia) - \#2, } \\
\text { Yoshihara et al. (2008) - \#4, Ito } \\
\text { et al. (2008) - \#4, Mueller et al. } \\
\text { (2008) - \#2 }\end{array}$ \\
\hline $\begin{array}{l}\text { Bekenov et al. } \\
\text { (1998) }\end{array}$ & $\begin{array}{l}\text { Lushchekina } \\
\text { et al. (1999), } \\
\text { Berger et al. } \\
(2008 b)\end{array}$ & & $\begin{array}{l}\text { Bekenov et al. } \\
\text { (1998), Lushchek- } \\
\text { ina et al. (1999), } \\
\text { Milner-Gulland et } \\
\text { al. (2001), Berger et } \\
\text { al. (2008a,b) }\end{array}$ & & $\begin{array}{l}\text { Berger et } \\
\text { al. } \\
(2008 b)\end{array}$ & $\begin{array}{l}\text { Bekenov et al. (1998) (Kaza- } \\
\text { khstan) - \#4, Lushchekina et al. } \\
(1999)(\text { Mongolia) - \#4, Baskin \& } \\
\text { Danell (2003)* - \#1 CMS } \\
(2006 a)^{*}-\# 4, \text { Wingard \& Zahler } \\
(2006)^{*}-\# 4, \text { Berger et al. } \\
(2008 a, b) \text { (Mongolia) - \#1 }\end{array}$ \\
\hline
\end{tabular}




\section{Northern Africa}

Blue Wildebeest (BW) Burchell's Zebra (BZ) Eland (E) Grant's Gazelle (G)

$X$ Hartebeest $(\mathrm{H})$ $\mathrm{Kob}(\mathrm{K})$ Mongalla Gazelle (M)

$X$ Scimitar Horned Oryx (O) Thompson's Gazelle (TG) Topi (T)

X Black Wildebeest (BkW) X Blesbok (B)

Blue Wildebeest (BW)

Burchell's Zebra (BZ)

Eland (E)

Hartebeest $(\mathrm{H})$

X Quagga (Q)

?X Springbok (S)

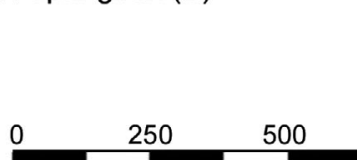

Africa

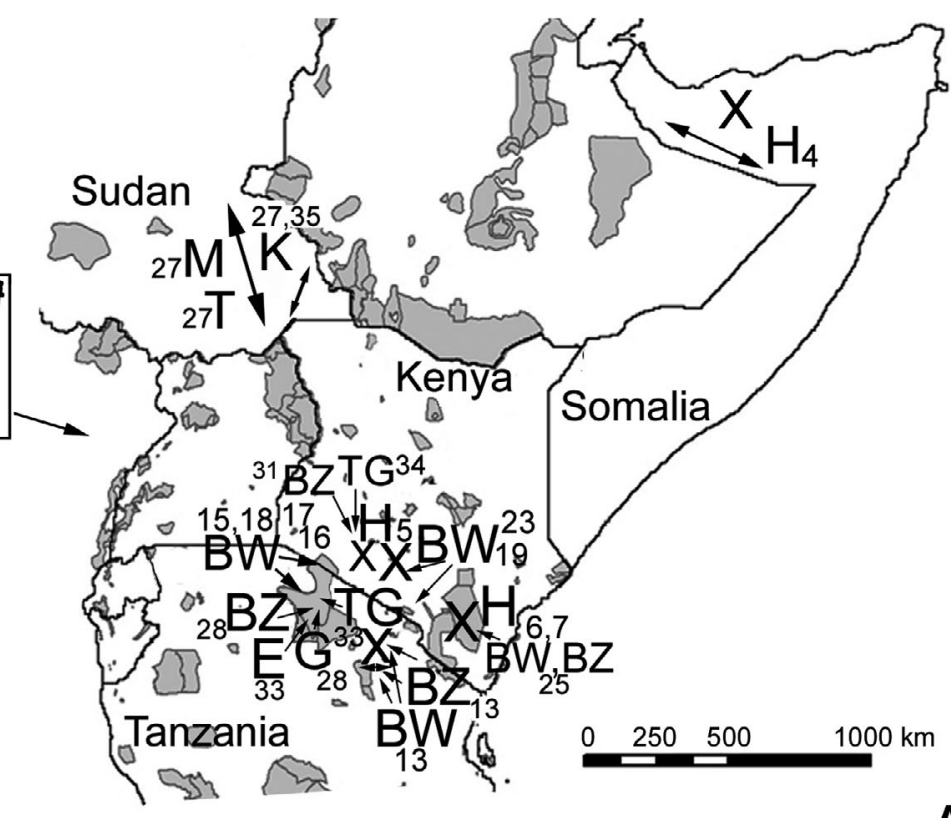

A

Fig. 1. Historic and current migrations in (A) northern and eastern Africa and (B) southern Africa for large mammals that move in aggregations. ' $\mathrm{X}$ ' in the legend indicates that the species is no longer migratory. ' $\mathrm{X}$ ' on the map shows where a species was historically migratory, but no longer is. Locations marked '?' represent migrations with an uncertain status. Lines with double arrows approximate locations with migratory routes. Lines with single arrows point to locations of current and historic migrations (for sprinkbok, these lines indicate 1-way movements. Numbers link to references and descriptions in Table 1. Light gray polygons are protected areas (WDPA Consortium 2005푼) 

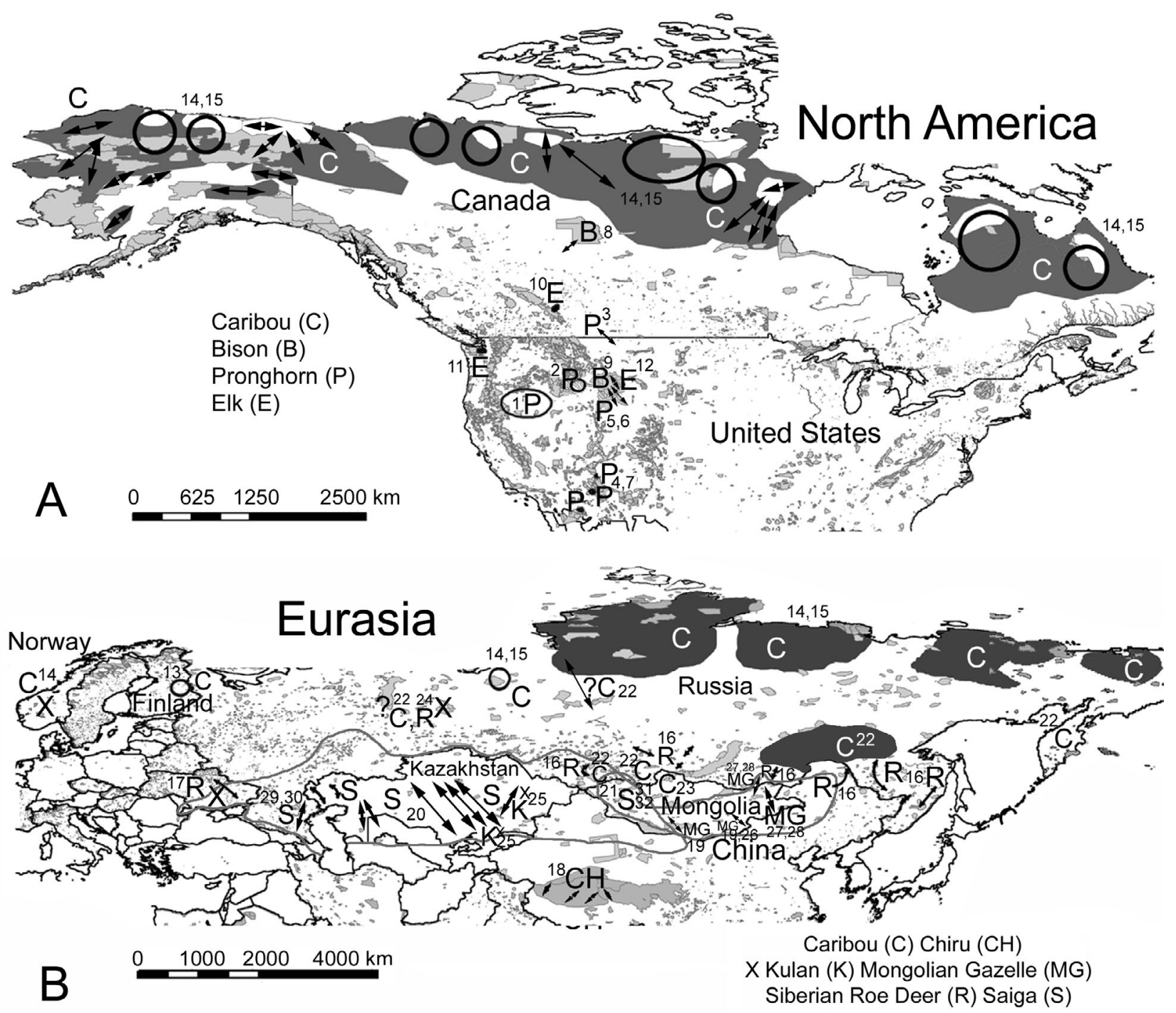

Fig. 2. Historic and current migrations in (A) North America and (B) Eurasia for large mammals that move in aggregations. Arrowed lines approximate locations of current and historic migrations. ' $\mathrm{X}$ ' represents an extinct mass migration, and black circles/ovals mark migrations with routes unknown or variable. Locations marked '?' represent migrations with an uncertain status. Gray, unfilled polygons outlining areas of Kazakhstan and Mongolia represent the historic migratory range for saiga and Mongolian gazelle, respectively. Dark gray, filled areas in conjunction with caribou depict migratory ranges, and white areas within them in (A) calving grounds. Numbers link to references and descriptions in Table 1. Light gray, filled polygons are protected areas (WDPA Consortium 2005).

(elk, caribou/reindeer, Siberian roe deer Capreolus pygargus, chiru). In tropical grasslands and savannas, the timing of rainfall is relatively predictable, but the actual distribution and amount of rainfall is patchy (Williamson et al. 1988, Boone et al. 2006). Therefore, migratory routes in tropical ecosystems remain unfixed, as migrants deviate to patches of young grass

${ }^{1}$ The WDPA is a joint product of UNEP and IUCN, prepared by UNEP-WCMC, supported by IUCN WCPA and working with Governments, the Secretariats of MEAs and collaborating NGOs. For further information: protectedareas@ unep-wcmc.org from local rain or fire events (Williamson et al. 1988, Mueller et al. 2008).

Many grassland migrants can gain adequate moisture from forage, e.g. blue wildebeest Conochaetes taruinus (Williamson et al. 1988), springbok (Skinner 1993), pronghorn (Ockenfels et al. 1994). However, during severe droughts, grass desiccates, and species seek surface water. This is especially marked for hartebeest Alcelaphus buselaphus, blue wildebeest, Burchell's zebra Equus burchellii, white-eared kob Kobus kob, saiga, kulan and pronghorn (Table 2).

When forage is abundant, migrants move less, when it is sparse, they move more (Williamson et al. 1988, 
Mduma et al. 1999). During severely dry years, when many migrants seek surface water, movement distances are at their maximum (Williamson et al. 1988). Numbers also affect movement distances. When wildebeest populations in the SME were reduced by rinderpest to $\sim 200000$ during the 1950s, their migratory routes were shorter than those of the current migration which comprises 1.3 million animals (Grzimek \& Grzimek 1960, Thirgood et al. 2004). This did not occur with changes in annual rainfall, indicating that movement distances were associated with animal numbers (Sinclair \& Norton-Griffiths 1979).

\section{Threats}

There are 2 principal ways to destroy a mass migration. First, kill a significant proportion of the migrants. Unsustainable hunting reduces migratory populations, contributing toward extirpation (e.g. scimitar horned oryx) and even extinction (e.g. quagga). Survivors may migrate shorter distances, or not at all, as migration distances decrease with lower numbers. If the population remains migratory, then they may no longer move in large masses, if such numbers no longer exist. Of the 20 mass migrants for which threats are listed, over-harvest is cited as a threat for 17 (Table 2).

The second principal threat is restricted access to food or water. This is an issue of habitat loss, which affects 17 of the mass migrants with listed threats (Table 2). Range can be taken directly, via agriculture, development (infrastructure, fossil fuels), or grazing livestock; fencing works indirectly, by barring access. Usually, agriculture and livestock secure the best lands, especially those which are productive during dry years. Several groups of migrants funnel into narrow corridors of 100 s of meters wide (geographical, topographical or human induced), making routes prone to amputation (migration 'bottlenecks') (Kahurananga \& Silkiluwasha 1997, Berger 2004, Berger et al. 2008a). Other migrants track traditional routes or ranges, and when access is denied or the range otherwise usurped, these migrants seem incapable of seeking essential resources elsewhere (Owens \& Owens 1983, Williamson et al. 1988, Toweill \& Thomas 2002). Transportation corridors (e.g. highways, railroads) often pose barriers for pronghorn, Mongolian gazelles, saiga and kulan (Ockenfels et al. 1994, Ito et al. 2005, 2006, Kaczensky et al. 2006, Berger et al. 2008b). Mass migrations usually extend beyond protected areas, which are simply too small to contain them. Hence, agriculture and development outside of parks often threaten migrations (Campbell \& Borner 1995, Kahurananga \& Silkiluwasha 1997, Homewood et al. 2001). Lack of adequate protection within parks also poses problems (Newmark 1987).
Climate change engenders longer-term threats. Concerns concentrate on migrants in higher latitudes where the pace and scale of habitat changes and the decoupling of climatic variables over disparate migratory ranges are highest, causing problems with mistimed migrations (Pulido 2007, Robinson et al. 2009). Migrants' abilities to adapt to changing environmental conditions are likely exacerbated by the other anthropogenic threats, such as habitat loss and fragmentation (Jetz et al. 2007)

\section{Conservation knowledge}

Africa, the continent with the most extinct and extant populations of migratory ungulates, has 17 reports containing information on the conservation of mass migrants, $60 \%$ of which occur at Step 1 or 2 of the conservation framework assessed in this study. The average is about 1 report per migrant; however, more than half refer to wildebeest. Five of these reports refer to the SME, the sole location to attain Step 6 in the framework. Three remaining mass migrants in Africa have no conservation-based information. There are 18 reports for Eurasia, half of which cover Step 4, but not necessarily Steps 1 to 3 . Siberian roe deer, chiru and kulan have only one report each describing any aspect of conservation. North America has the most advanced information (with fewest migrants), and 10 out of 16 reports reaching Step 3 or above (Table 2). The only North American efforts advancing to Step 5 are those for bison and pronghorn in the Greater Yellowstone Ecosystem (GYE).

\section{Extinct migrations}

Reducing migrants by over-harvest or removing range contributes to population declines, lowers the distances migrants travel, and can destroy the migration and species. In Africa, migrations of scimitar horned oryx and hartebeest have disappeared from the Sahara of Niger and Chad, and the Ogaden of Somalia and Ethiopia respectively (East 1988, Mallon $\&$ Kingswood 2001). Wildebeest migrations in the AthiKapiti Plains in southeast Kenya are extinct (J. Oguto pers. comm.). Wildebeest once migrated northeast of Etosha National Park in Namibia during dry seasons, but cordon fencing in 1973 closed all movements (Berry 1997); fencing, coupled with harvest on farms and communal lands, caused numbers to fall from 30000 in 1964 to 2000 in 1993 (Gasaway et al. 1996). Burchell's zebra also declined by about 20000 from 1953 to 1991 (Gasaway et al. 1996). Fencing in Kruger National Park in South Africa blocked wildebeest 
migration, and populations declined from 6000 to 750 (Braack 1973, Whyte \& Joubert 1988). Historical (1850s) migrations of 10000 s of trekbokke (springbok, black wildebeest, blesbok, eland and quagga), no longer occur in the Karoo and Highveld of South Africa (Skinner \& Chimimba 2005) and quagga are extinct as a species (Hack et al. 2002). Reports of extant mass migrations of springbok in Botswana are unconfirmed (Skinner \& Louw 1996).

Reports describe mass migrations of Siberian roe deer up to the second half of the 19th century in the Ukraine, but these migrations are presumed lost, largely from over-harvesting (Kirikov 1959, Baskin \& Danell 2003). Many details of Siberian roe deer migrations in Siberia are unclear (Danilkin et al. 1992, Danilkin 1996). Kulan reportedly migrated from NW Kazakhstan to Betpakdala in the southeast, but these regular migrations disappeared as kulan numbers dropped (Baskin \& Danell 2003). The present status of kulan in Asia Minor countries such as Iran remains unclear. Saiga migrations used to be far more widespread up to the 18th century, going as far west as Romania, present-day Macedonia, and western Ukraine (Lushchekina \& Struchkov 2001). Similarly, migrations of Mongolian gazelle were formerly common throughout Mongolia and parts of Russia and China (Jiang et al. 1998).

19th century hunters nearly exterminated migratory bison occupying the expansive grasslands of central North America (Dary 1974). Only 2 remnant populations of migratory bison remain, one in Yellowstone National Park in the USA and the other in Wood Buffalo National Park in Canada (Meagher 1973, Van Vuren \& Bray 1986, Gates et al. 2001).

\section{Threatened migrations}

Many African ecosystems contain guilds of extant migratory species; we summarized these from north to south. Mass migrations of kob, tiang Damaliscus lunatus and mongalla gazelle Gazella thomsonii albonotata occur in the Boma-Jonglei Ecosystem in southern Sudan (Fryxell \& Sinclair 1988b, East 1999). Many believed these migrations decimated by civil war, but recent surveys (2007) indicate $\sim 1$ million migratory ungulates in and adjacent to Boma National Park (www.msnbc.msn.com/id/19191023/?GT1=10056).

Mass migrations of wildebeest, Burchell's zebra, Grant's gazelle Nanger granti, Thomson's gazelle Gazella thomsonii and eland persist in the SME of Tanzania and Kenya (Sinclair \& Arcese 1995, Thirgood et al. 2004). A separate population of wildebeest migrates from the Masai Mara National Reserve north to the Loita Plains. This population has declined from $\sim 100000$ to $\sim 25000$ over 20 years (late 1970s to late 1990s) because of habitat loss on the Loita Plains (Homewood et al. 2001, Ottichilo et al. 2001). Wildebeest, zebra and Grant's gazelle also migrate in the Tarangire Ecosystem, $200 \mathrm{~km}$ southeast of the SME (Borner 1985, Kahurananga \& Silkiluwasha 1997). Migration routes to the west and north of Tarangire National Park are blocked by agriculture and there is considerable poaching pressure outside the park. Wildebeest populations declined from $\sim 50000$ to 5000 between 1988 and 2001 (TAWIRI 2001).

Populations of wildebeest and zebra migrate in masses between Liuwa Plains National Park (Zambia) and Kameia National Park (Angola). This migration is largely undocumented. Wildebeest and zebra also migrate in aggregations within the Chobe-Linyanti Ecosystem in northern Botswana (also largely undocumented; N. Owen-Smith pers. comm.). Severe drought in the 1980 s killed many migratory eland, hartebeest $(\sim 10000)$ and wildebeest ( 80 000) in the Kgalagadi Transfrontier Park and the Central Kalahari Game Reserve in southern Botswana, as livestock use and veterinary fences block access to dry season refuges (forage and water) outside protected areas (Owens \& Owens 1983, Spinage 1992, Verlinden 1998, C. Thouless unpubl. data, R. Brett pers. comm.); some 50000 wildebeest starved around Lake Xau in 1983 alone (Williamson \& Mbano 1988). Populations of non-migratory ungulates in the Kalahari have not shown similar declines.

Eurasia contains 2 subspecies of saiga: Saiga tatarica tatarica (mainly in Kazakhstan and Russia) and S. $t$. mongolica (in Mongolia). S. t. tatarica populations have collapsed (Milner-Gulland et al. 2001, CMS 2006a), with habitat loss and overhunting likely responsible for dividing remaining animals into 4 migratory populations (CMS 2006a). Recent studies have assessed the status and movements of $S$. $t$. mongolica in Mongolia (Berger et al. 2008a,b). This subspecies has also severely declined in numbers, and remaining migrations are threatened by increasing anthropogenic pressures.

Chiru migrate in the Chang Tang National Park on the Tibetan Plateau (Schaller 1998, Lian et al. 2005) and Mongolian gazelle migrate 1000s of kilometers across the steppes of Mongolia, China and Russia (Ito et al. 2005, 2006, Mueller et al. 2008). Both species were decimated by hunting during the 20th century, with chiru recently stabilizing at 100000 (http://news. nationalgeographic.com/news/2007/02/070206-tibetantelope.html) and current estimates of Mongolian gazelle of $\sim 800000$ (Olson et al. 2005). Some data on Mongolian gazelle movements are now available and suggest that populations may be more nomadic than migratory, which may make their conservation more difficult (Mueller et al. 2008). 
Caribou/reindeer migrate in at least 13 populations in Alaska and Canada and at least 9 populations in Russia (Baskin \& Danell 2003, Ulvevadet \& Klokov 2004). Herds move between calving areas in the tundra and wintering areas in the taiga and migratory routes may change between years (Fancy et al. 1989, Baskin \& Danell 2003, Ulvevadet \& Klokov 2004). Some populations have declined whereas others have increased (Ulvevadet \& Klokov 2004, www.rangifer. net/carma/). Increasing oil and gas exploitation threaten reindeer migrations on the Russian Taymyr peninsula and in southern Yakutia (L. Baskin pers. comm.).

Pronghorn and elk in western North America often display fidelity to migratory routes and winter range (Rudd et al. 1983, Sawyer et al. 2002, Toweill \& Thomas 2002). Increases in fences and human habitation can shorten, impede and block these migrations (Ockenfels et al. 1994, Berger 2004). We did not find any information regarding mass migrations of the Eurasian red deer Cervus elaphus.

\section{DISCUSSION}

The principal conclusion drawn from this global audit is that mass migrations of mammals are under major threat throughout the world. Migrations of 6 of these mammals have been completely eradicated, and nearly all have lost some aggregated migrations. These are undoubtedly underestimates, given that reporting is poor, and other migrations probably existed in areas where humans now dominate landscapes. The main causes are unsustainable hunting and loss of seasonal ranges and/or migration routes through fencing, livestock, agriculture or human settlement. A number of migrations cross international borders, so solutions to mitigate threats rely on creative schemes often spanning countries and diverse cultures on site. Rarely has such work been done, and rarer still have efforts taken hold (Table 2).

Because the same factors threaten most migrations, the solutions for protecting them in a given location assist with conserving migrations elsewhere. Examples from 2 disparate migratory ecosystems, the SME and GYE, show that, given suitable commitment from governments, conservationists and scientists, conserving mass migrations is not an unrealistic ideal.

The SME is the world's largest ( 2 million migrants) and most species-diverse extant migration (Sinclair \& Arcese 1995, Thirgood et al. 2004). It may be the only ecosystem where migratory populations increased during the 20th century - in this case because of the eradication of rinderpest in the 1950s. Migratory populations are currently maintained through the protec- tion of an intact ecosystem. The annual range for the migrants is largely contained within the protected area network, including wet and dry season ranges and transition zones (Thirgood et al. 2004). The entire range is without fences. Although illegal hunting removes $\sim 40000$ wildebeest each year, this harvest is sustainable and the population is limited by food availability in the dry season (Mduma et al. 1999). Intensive activities by management authorities limit poaching (Hilborn et al. 2006). Ecotourism finances these activities, which are reinforced by long-term commitments from international conservation organizations. Maintaining these protected areas receives government support because of ecotourism's role in development (Thirgood et al. 2008). No other ecosystem containing migratory ungulates shares all of these attributes and it is perhaps no coincidence that the SME is the global flagship for successful conservation of migratory ungulates.

The GYE contains the longest and most diverse ungulate migrations in North America (Berger 2004). These migrations remain largely unprotected, with highways, housing, fencing, and energy extraction sites impeding movements both inside and outside protected areas (Berger 2004, Sawyer et al. 2005). Research projects focus on ungulate movements (routes, bottlenecks) to determine what constitutes migratory range and where this range is located, and establish population objectives to maintain and conserve these migrations (Berger et al. 2006). Solutions require implementing conservation plans far beyond protected area boundaries, such as purchasing conservation easements and reducing surface impacts to public lands, especially during migratory periods. Here and elsewhere, migration corridors can facilitate the movement of large mammal populations. For example, eland re-colonized Lake Manyara National Park (Newmark 1996) and projects envision elephants migrating between transboundary parks in southern Africa (van Aarde \& Jackson 2007). As such, the myriad of conservation measures required to protect migrants in the largely unprotected GYE presents a marked contrast to the protected area-based conservation strategy in the SME.

\section{Aggregated migrations vs. other types of movement}

Many migrations are poorly known, which complicates abilities to distinguish between aggregated migrations and other forms of movement. Some migrations composed of relatively few individuals could represent disaggregated movements, or be remnants of mass migrations. Consequently, our list could be considered too inclusive if it included species that behave 
nomadically rather than as migrants. Alternatively, our collection could be considered restrictive. Elephants Loxodonta africana, for example, were excluded because there lacks literature describing their mass migrations, and experts state that they do not migrate in aggregations (I. Douglas-Hamilton \& R. van Aarde, pers. comm.). Another view considers that elephants do make aggregated migrations; however, movements are widely spaced and coordinated through longdistance communication. (N. Owen-Smith pers. comm.).

Similar difficulties exist in defining other African ungulates. For example, Grevy's zebra Equus grevyi move in response to rainfall in northern Kenya, but such behavior is not a mass migration (Williams 2002, S. Williams pers. comm.). Lechwe Leche leche have been reported as migratory in the Chobe-Linyanti Ecosystem in Botswana (Child \& von Richter 1969). Here, as elsewhere, lechwe inhabit floodplains and track seasonal changes in water levels. For example, 40 000 lechwe aggregate on $100 \mathrm{~km}^{2}$ of floodplain at Bangweulu in Zambia during the wet season (Thirgood et al. 1994). These aggregations dissolve in the dry season, as small groups disperse into permanent swamps. Hence, we do not consider such lechwe populations to migrate in masses.

In Eurasia, the goitered gazelle Gazella subgutturosa ranges from Mongolia and northwestern China to Israel and the Arabian Peninsula, and is highly nomadic throughout this range. We excluded this species, since clear references to mass migrations are lacking. However, few studies explore the spatial distribution of this species, and some populations could have fixed summer and winter ranges. Moreover, goitered gazelle have declined rapidly in numbers with $>90 \%$ population reduction in many of their range states from the 1940s to 1980s (Mallon \& Kingswood 2001), perhaps causing the disintegration of migrations into nomadic behavior. Goitered gazelle in central Asia seem to aggregate in winter and used to migrate $450-700 \mathrm{~km}$, but due to reduced population sizes, the distances declined to 50-60 km (Baskin \& Danell 2003). Other Eurasian and African species display similar behavior, and some may have migrated in masses in the past but no longer do (e.g. Bactrian camel Camelus bactrianus, Przewalski's horse Equus caballus, Tibetan gazelle Procapra picticaudata, addax Addax nasomaculatus and dama gazelle Nanger dama).

The recent study by Mueller et al. (2008) illustrates the fine border between migratory and nomadic behavior. The authors show that Mongolian gazelle in the eastern steppes of Mongolia display irregular seasonal movement patterns, escaping snow in winter and tracking vegetation quality in summer. This species, or at least this subpopulation, appears more nomadic, and less migratory, than earlier studies suggest. This begs the question whether or not the difference between nomadic and migratory behavior is an inherent species trait or whether it is driven by the regularity of climatic variation, as this example suggests. Other large-scale migrations, such as migrations in the SME, occur in regional areas with greater predictability in the timing and spatial patterns of rainfall (though highly variable at local scales). Future climate change might severely reduce this predictability and, hence, populations that we currently define as migratory could increasingly become nomadic. While in this paper we aimed at providing an audit of mass migrants using fixed ranges, obviously all migrants are worthy of conservation, irrespective of their movement patterns.

The fact that South America and Australia lack mammals that migrate in aggregations poses interesting ecological questions. For South America, increases in forest cover at the expense of grasslands during the mid-Holocene likely contributed to the loss of largebodied mammalian fauna and migrations (de Vivo \& Carmignotto 2004). The only large-bodied, migratory mammal known from South America is the guanaco Lama quanicoe, which makes altitudinal shifts across seasons (Nowak 1999). New work may reveal that these animals make aggregated migrations (The Patagonia Times 2008). Australia lost $43 \%$ of large bodied, herbivorous mammals ( $>5 \mathrm{~kg}$ ) during the Pleistocene, compared to $13.5 \%$ in Africa (OwenSmith 1987). This outcome likely affected the potential number of large-bodied mammals to migrate on the Australian continent.

\section{Gray literature}

This project reports facts from gray literature which we judged credible. Although it is not universally available or evaluated by peer review, we considered gray literature for 2 reasons. First, sometimes gray sources represent the best and only available knowledge on these migratory species and ecosystems. Second, we must learn and adapt using the best available information we have to inform society of the concern, and work toward maintaining the migrations that remain. For example, would it be better to report that kob migrations in Sudan are extinct based on older, peer-reviewed publications, rather than present recent, unpublished surveys stating otherwise? Certainly, peer-reviewed science should remediate the deficiencies, advance the understanding of these migrations, and monitor the effectiveness of conservation strategies aimed at protecting them. Our synthesis may also have missed important aspects of the literature that is difficult to access via standard scientific 
search engines, specifically literature from the vast Russian territory. In addition, data from the Asia Minor region, including countries such as Uzbekistan, Iran and Afghanistan, is severely deficient. These countries host potentially migrating species, such as goitered gazelle and kulan.

\section{Veterinary policy}

An increasing threat to animal migrations is that of international veterinary policy. We identify 2 main issues, the first being veterinary cordon fences. Since the late 1950s, these have been erected in Southern Africa to separate livestock from wildlife populations. These fences block migration routes and have devastating effects on ungulate populations (Owens \& Owens 1983, Williamson et al. 1988, Martin 2005, Mbaiwa \& Mbaiwa 2006). Their purpose is to limit disease transfer from wild to domestic ungulates in order to meet the high standards of disease management put forward by beefimporting nations (Taylor \& Martin 1987, Martin 2005, Mbaiwa \& Mbaiwa 2006). Ironically, the transfer of exotic diseases from domestic to wild populations is an increasing threat, and migratory ungulates might be especially sensitive due to their gregarious behavior, as likely exemplified by Mongolian gazelle (Lhagvasuren \& Milner-Gulland 1997, Nyamsuren et al. 2006). Despite any clear evidence that these fences effectively control disease outbreaks, there are rising concerns that this method will be copied elsewhere, and hence threaten other migrations (e.g. Mongolia; Nyamsuren et al. 2006). The second issue is culling to control disease transfer to domestic stock. This policy has reduced migratory populations in the past (Newmark 2008), and threatens existing migratory populations now (e.g. Mongolian gazelle; Nyamsuren et al. 2006). We recognize the needs to control the spread of potentially dangerous zoonotic diseases; however, we seek novel solutions that retain ecological processes, such as mass migrations.

\section{Conserving mass migrants}

The Convention on Migratory Species works internationally to conserve migrations across taxa (www. cms.int/). They focus on species threatened with extinction, but include other species whose migrations would significantly benefit from international cooperation, including strictly migratory and nomadic species. The Convention currently lists 3 large mammals that migrate(d) in aggregations, the scimitar-horned oryx (Extinct in the Wild), Saiga tatarica tatarica (Critically Endangered), and the Mongolian gazelle (Least Con- cern) (IUCN 2008). Clear criteria for listing are absent. We also note that endangered chiru is unlisted (IUCN 2008). Other aggregated migrants, with migrations no longer existing, or with migrations declining faster than efforts to understand or conserve them, also remain absent (Table 2). We encourage this group (and others) to advance beyond species-level conservation planning to include preserving the migratory phenomenon itself (see also Wilcove \& Wikelski 2008). Migratory behavior for these species can cease (and for some it has, Table 1) without species going extinct. Relegating these wide-ranging migrants to small zoos and parks because of poaching and extensive habitat loss from agriculture or livestock is inadequate on ecological and ethical grounds (e.g. ecosystem collapses and loss of the intrinsic ecology of such species). The Convention represents an international mechanism for change, and we urge their efforts in making changes happen.

Conserving mass migrants means preserving animals' freedom of movement in response to the temporal aspects of forage across seasonal extremes. This requires understanding basic parameters of the migration (e.g. location, numbers, routes, distances traveled), ecological drivers, habitat needs and threats. When migrants are excluded from forage and water resources , their numbers plummet and migrations disappear. Migrants remain at low population levels in small areas that have enough resources to maintain them.

This synthesis reviews the information currently available and pertinent for the conservation of mass migrations and highlights gaps to focus scientific attention. When addressed, proven methods such as protecting seasonal ranges, removing barriers, promoting ecotourism, securing long-term support from governments and NGOs, mitigating incompatible land uses and garnering conservation easements, can preserve the ecological, economic and aesthetic values of aggregated migrations.

Acknowledgements. We thank M. Borner, T. Brooks, J. du Toit, E. Dinerstein, R. Estes, R. Guildemond, N. Horning, P. Kaczensky, A. Lushchekina, E. J. Milner-Gulland, J. Morrison, N. Owen-Smith, K. Redford, E. Sanderson, A. R. E. Sinclair and E. Sterling for advice and assistance. Financial support came from the John D. and Catherine T. MacArthur Foundation, Frankfurt Zoological Society, the Wildlife Conservation Society, and the Marie Curie Transfer of Knowledge project BIORESC within the European Commission's 6th Framework Programme (Contract no. MTKD-CT-2005029957).

\section{LITERATURE CITED}

Baskin L, Danell K (2003) Ecology of ungulates: a handbook of species in eastern Europe and northern and central Asia. Springer, Berlin 
Bekenov AB, Grachev Iu A, Milner-Gulland EJ (1998) The ecology and management of the saiga antelope in Kazakhstan. Mamm Rev 28:1-52

Berger J (1991) Greater Yellowstone's native ungulates: myths and realities. Conserv Biol 5:353-363

Berger J (2004) The last mile: how to sustain long-distance migration in mammals. Conserv Biol 18:320-331

Berger J, Cain SL, Berger K (2006) Connecting the dots: an invariant migration corridor links the Holocene to the present. Biol Lett 2:528-531

Berger J, Young JK, Berger KM (2008a) Protecting migration corridors: challenges and optimism for Mongolian saiga. PLoS Biol 6:e165-167

Berger J, Berger KM, Bergen S, Buuveibaatar B and others (2008b) Migration bottlenecks, climate, and the conservation of Pleistocene relicts in central Asia. Open Conserv Biol J 2:9-10

Berry HH (1980) Behavioral and eco-physiological studies on blue wildebeest (Conochaetes taruinus) at the Etosha National Park. PhD thesis, University of Cape Town

Berry HH (1997) Aspects of wildebeest (Connochaetes taurinus) ecology in Etosha National park: a synthesis for future management. Madoqua 20:137-148

Bonifica (1993) Technical Assistance to the Central Kalahari Borehole Project. Department of Wildlife and National Parks, Gabarone

Boone RB, Thirgood SJ, Hopcraft JGC (2006) Serengeti wildebeest migratory patterns modeled from rainfall and new vegetation growth. Ecology 87:1987-1994

Borner M (1985) The increasing isolation of Tarangire National Park. Oryx 19:91-96

Boudreau TA (2003) Units 19 and 21 caribou management report. In: Healy C (ed) Caribou management report of survey and inventory activities 1 July 2000-30 June 2002. Alaska Department of Fish and Game, Juneau, AK, p 134-147

Braack HH (1973) Population dynamics of the blue wildebeest (Connochaetes taurinus taurinus Burchell, 1823), in the Central District of the Kruger National Park. Project report, University of Rhodesia, Salisbury

Bradley RM (1977) Aspects of the ecology for the Thomson's gazelle in the Serengeti National Park, Tanzania. PhD dissertation, Texas A\&M University, College Station, TX

Bright JL, Van Riper C III (2000) Pronghorn home ranges, habitat selection, and distribution around water sources in Northern Arizona. Tech Rep Ser USGSFRESC/COPL/2000 18, US Geological Service, Denver, CO

Brown C (1985) The Sand Creek elk: Population status, movements, and distribution. Project No. W-160 R, Job Completion Report, Idaho Department of Fish Game, Boise, ID

Campbell K, Borner M (1995) Population trends and distribution of Serengeti herbivores: implications for management. In: Sinclair ARE, Arcese P (eds) Serengeti II: Dynamics, management and conservation of an ecosystem. University of Chicago Press, Chicago, IL, p 117-145

Caughley G (1976) Wildlife management and the dynamics of ungulate populations. In: Coaker TH (ed) Applied biology, Vol 1. Academic Press, New York, p 183-246

Child G, LeRiche JD (1969) Recent springbok treks (mass movements) in southwestern Botswana. Mammalia 33:499-504

Child G, von Richter W (1969) Observation on ecology and behavior of lechwe, puku and waterbuck along the Chobe River, Botswana. Z Saugetierkd 34:275-295

Clark EL, Munkhbat J, Dulamtseren S, Baillie JEM, Batsaikhan N, Samiya R, Stubbe M (2006a) Mongolian Red List of mammals. Regional Red List Series, Vol 1. Zoological Society of London, London. Available at: www.
zoo.cam.ac.uk/ioz/projects/MongolianRedList_mammals. pdf (accessed 4 Oct 2008)

Clark EL, Munkhbat J, Dulamtseren S, Baillie JEM and others (2006b) Summary Conservation Action Plans for Mongolian Mammals. Regional Red List Series, Vol 2. Zoological Society of London, London. Available at: www.zoo.cam. ac.uk/ioz/projects/MongolianActionPlan_mammals.pdf (accessed 4 Oct 2008)

Convention on Migratory Species (2006a) Report of the first meeting of the signatories to the memorandum of understanding concerning conservation, restoration and sustainable use of the Saiga antelope (Saiga tatarica tatarica). Annex 5, Revised Overview Report. Convention on Migratory Species, Almaty. Available at: www.cms.int/species/ saiga/post_session/Annex_05_Revised_Overview_Report_E. pdf (accessed 4 Oct 2008)

Convention on Migratory Species (2006b) Sahelo-Saharan antelopes: status and perspectives. CMS Tech Ser Publ No. 11, CMS, Bonn

Craighead JJ, Atwell G, O'Gara BW (1972) Elk migration in and near Yellowstone National Park. Wildl Monogr 29

Danilkin AA, Darman YuA, Minayev AN (1992) The seasonal migrations of a Siberian roe deer population. Rev Ecol Terre Vie 47:231-243

Danilkin A (1996) Behavioral ecology of Siberian and European roe deer. Wildlife Ecology and Behavior Series 2. Chapman \& Hall, London

Dary DA (1974) The buffalo book: the full saga of the American animal. Sage Books, Chicago, IL

de Vivo M, Carmignotto AP (2004) Holocene vegetation change and the mammal faunas of South America and Africa. J Biogeogr 31:943-957

Deblinger RD (1980) Ecology and behavior of pronghorn in the Red Desert, Wyoming with reference to energy development. PhD dissertation, Colorado State University, Fort Collins, $\mathrm{CO}$

Deshmukh IK (1984) A common relationship between precipitation and grassland peak biomass for east and southern Africa. Afr J Ecol 22:181-186

Dingle H (1996) Migration: the biology of life on the move. Oxford University Press, Oxford

East R (1988) African antelope: status survey and conservation action plan, Parts 1-3. IUCN/Species Survival Commission African Antelope Specialist Group, Gland

East R (1999) African Antelope Specialist Group: African antelope database 1998. Occasional Paper 21, IUCN, Gland

Ebedes H (1970) The nomadic plains zebra Equus burchelli antiquorum H Smith 1941 of the Etosha Salina. Report Director, Nature Conservation, Windhoek

Eregdendagvaa D (1954) Saiga antelope in Mongolian People's Republic. News Irkutsk Agric Inst 6:333-343

Estes RD (1991) The behavior guide to African mammals. University of California Press, Berkeley, CA

Fancy SG, Pank LF, Whitten KR, Regelin WL (1989) Seasonal movements of caribou in arctic Alaska as determined by satellite. Can J Zool 67:644-650

Foster J, Kearney D (1967) Nairobi National Park game census, 1966. East Afr Wildl J 5:112-120

Frank DA, McNaughton SJ (1992) The ecology of plants, large mammalian herbivores and drought in Yellowstone National Park. Ecology 73:2043-2058

Frank DA (1998) Ungulate regulation of ecosystem processes in Yellowstone National Park: direct and feedback effects. Wildl Soc Bull 26:410-418

- Fryxell JM, Sinclair ARE (1988a) Causes and consequences of migration by large herbivores. Trends Ecol Evol 3:237-241 
Fryxell JM, Sinclair ARE (1988b) Seasonal migration by white-eared kob in relation to resources. Afr J Ecol 26: $17-31$

Fryxell JM, Wilmshurst JF, Sinclair ARE (2004) Predictive models of movement by Serengeti grazers. Ecology 85:2429-2435

$>$ Fryxell JM, Wilmshurst JF, Sinclair ARE, Haydon DT, Holt RD, Abrams PA (2005) Landscape scale, heterogeneity and the viability of Serengeti grazers. Ecol Lett 8:328-335

Gardner CL (2003) Units 20 and 25 caribou management report. In: Healy C (ed) Caribou management report of survey and inventory activities 1 July 2000-30 June 2002. Alaska Department of Fish and Game, Juneau, AK, p 160-189

Gasaway WC, Gasaway KT, Berry HH (1996) Persistent low densities of plain's ungulates in Etosha National Park, Namibia: testing the food regulation hypothesis. Can J Zool 74:1556-1572

Gates CC, Mitchell J, Wierzchowski J, Giles L (2001) A landscape evaluation of bison movements and distribution in northern Canada. AXYS Environmental Consulting, Calgary

Grzimek M, Grzimek B (1960) A study of the game of the Serengeti Plains. Z Saugetierkd 25:1-61

Hack MA, East R, Rubenstein DL (2002) Equus quagga. 2004 IUCN Red List Threatened Species, IUCN, Gland. Available at: www.iucnredlist.org/ (accessed 15 Feb 2005)

Hanley TA (1982) The nutritional basis for food selection by ungulates. J Range Manag 35:146-151

Hilborn R, Arcese P, Borner M, Hando J and others (2006) Effective enforcement in a Conservation Area. Science 314:1266

Hoskinson RL, Tester JR (1980) Migration behavior of pronghorn in southeastern Idaho. J Wildl Manag 44:132-144

Homewood $\mathrm{K}$, Lambin EF, Coast E, Kariuki A and others (2001) Long-term changes in Serengeti-Mara wildebeest and land cover: pastoralism, population or policies? Proc Natl Acad Sci USA 98:12544-12549

Houston DB, Schreiner EG, Moorhead BB, Krueger KA (1990) Elk in Olympic National Park: Will they persist over time? Nat Areas J 10:6-11

Ito TY, Miura N, Lhagvasuren B, Enkhbilig D, Takatsuki S, Tsunekewa A, Jiang Z (2005) Preliminary evidence of a barrier effect of a railroad on the migration of Mongolian gazelle. Conserv Biol 19:945-948

Ito TY, Miura N, Lhagvasuren B, Enkhbilig D, Takatsuki S, Tsunekewa A, Jiang Z (2006) Satellite tracking of Mongolian gazelle (Procarpa gutturosa) and habitat shifts in their seasonal ranges. J Zool (Lond) 269:291-298

Ito TY, Okada A, Buuveibaatar B, Lhagvasuren B, Takatsuki S, Tsunekawa A (2008) One-sided barrier impact of an international railroad on Mongolian gazelles. J Wildl Manag 72:940-943

IUCN (2008) 2008 IUCN Red List of Threatened Species. IUCN, Gland. Available at: www.iucnredlist.org (accessed 15 Oct 2008)

$>$ Jetz W, Wilcove DS, Dobson AP (2007) Projected impacts of climate and land-use change on the global diversity of birds. PLoS Biol 5:e157

Jiang Z, Takatsuki S, Gao Z, Jin K (1998) The present status, ecology and conservation of the Mongolian gazelle, Procapra gutturosa: a review. Mamm Study 23:63-78

Joly DO, Messier F (2001) Limiting effects of bovine brucellosis and tuberculosis on wood bison within Wood Buffalo National Park. Final report April 2001. University of Saskatchewan, Saskatoon, SK

Kaczensky P, Sheehy DP, Walzer C, Johnson DE, Lkhagva- suren D, Sheehy CM (2006) Room to roam? The threat to kulan (wild ass) from human intrusion. Mongolia Discussion Papers, East Asia and Pacific Environment and Social Development Department, The World Bank, Washington, DC. www.worldbank.org/eapenvironment

- Kahurananga J (1981) Population estimates, densities and biomass of large herbivores in Simanjiro Plains, Northern Tanzania. Afr J Ecol 19:225-238

Kahurananga J, Silkiluwasha F (1997) The migration of zebra and wildebeest between Tarangire National Park and Simanjiro Plains, northern Tanzania, in 1972 and recent trends. Afr J Ecol 35:179-185

Kirikov SV (1959) Changes in the fauna of geographical zones of the USSR, the 13th to 19th centuries: the steppe zone and forest steppe. Academy of Sciences Publishing House, Moscow

Larter NC, Sinclair ARE, Ellsworth T, Nishi J, Gates CC (2000) Dynamics of reintroduction in an indigenous large ungulate: the wood bison of northern Canada. Anim Conserv 3:299-309

- Leimgruber P, McShea WJ, Brookes CJ, Bolor-Erdene L, Wemmer C, Larson C (2001) Spatial patterns in relative primary productivity and gazelle migration in the eastern steppes of Mongolia. Biol Conserv 102:205-212

Lhagvasuren B, Milner-Gulland EJ (1997) The status and management of the Mongolian gazelle (Procapra gutturosa) population. Oryx 31:127-134

Lian XM, Su JP, Zhang TZ, Cao YF (2005) The characteristics of social groups of the Tibetan antelope in the Kekexili region. Acta Ecol Sin 25:1341-1346

Lian XM, Zhang TZ, Cao YF, Su JP, Thirgood SJ (2007) Group size effects foraging and vigilance in migratory Tibetan antelope. Behav Processes 76:192-197

Lushchekina AA, Neronov VM, Amgalan L, Dulamtseren S (1999) The status and prospects for conservation of the Mongolian saiga, Saiga tatarica mongolica. Oryx 33:20-29

Lushchekina A, Struchkov A (2001) The saiga antelope in Europe: once again on the brink? The Open Country 3: 11-24. Available at: www.saigak. biodiversity.ru/publications/open_country02.pdf (accessed 4 Oct 2008)

Mallon DP, Kingswood SC (2001) Antelopes, Part 4: North Africa, the Middle East, and Asia. Global Survey and Regional Action Plans. Species Survival Commission Antelope Specialist Group, IUCN, Gland

Martin RB (2005) The Influence of veterinary control fences on certain wild large mammal species in the Caprivi, Namibia. In: Osofsky SA, Cleaveland S, Karesh WB, Kock MD, Nyhus PJ, Starr L, Yang A (eds) Conservation and development interventions at the wildlife/livestock interface: implications for wildlife, livestock and human health. Occasional Paper of the IUCN Species Survival Commission No. 30, IUCN, Gland, p 27-39

Mbaiwa JE, Mbaiwa OI (2006) The effects of veterinary fences on wildlife populations in Okavango Delta, Botswana. Int $\mathrm{J}$ Wilderness 12:17-23. Available at: www. wilderness.net/library/documents/IJWDec06_Mbaiwa.pdf (accessed 2 Feb 2009)

McDonald JN (1981) North American bison: their classification and evolution. University of California Press, Berkeley

Mduma SAR, Sinclair ARE, Hilborn R (1999) Food regulates the Serengeti wildebeest migration: a 40 -year record. J Anim Ecol 68:1101-1122

Meagher M (1973) The bison of Yellowstone National Park. National Park Service, Washington DC 1:1-161

Meagher M (1989) Range expansion by bison of Yellowstone National Park. J Mammal 70:670-675

McNaughton SJR (1985) Ecology of a grazing ecosystem: the 
Serengeti. Ecol Monogr 55:259-294

McNaughton SJR, Ruess W, Seagle SW (1988) Large mammals and process dynamics in African ecosystems. BioScience 38:794-800

Milner-Gulland EJ, Kholodova MV, Bekenov A, Bukreeva OM, Grachev Iu A, Amgalan L, Lushchekina AA (2001) Dramatic decline in saiga antelope populations. Oryx 35:340-345

Mitchell GJ (1980) The pronghorn antelope in Alberta. Gulf Canada Resources, and Alberta Department of Energy and Natural Resources Fish and Wildlife Division, Edmonton

Morgantini LE, Hudson RJ (1988) Migratory patterns of the wapiti Cervus elaphus, in Banff National Park, Alberta. Can Field Nat 102:12-19

$>$ Morrison JC, Sechrest W, Dinerstein E, Wilcove DS, Lamoreux JF (2007) Persistence of large mammal faunas as indicators of global human impacts. J Mammal 88: 1363-1380

Mueller T, Olsen KA, Fuller TK, Schaller GB, Murray MG, Leimgruber $P$ (2008) In search of forage: predicting dynamic habitats of Mongolian gazelles using satellitebased estimates of vegetation productivity. J Appl Ecol 45:649-658

Murray MG (1995) Specific nutrient requirements and migration of wildebeest. In: Sinclair ARE, Arcese P (eds) Serengeti II: Dynamics, management and conservation of an ecosystem. University of Chicago Press, Chicago, IL, p 231-256

Murray MG, Brown D (1993) Niche separation of grazing ungulates in the Serengeti - an experimental test. J Anim Ecol 62:380-389

Newmark WD (1987) A land-bridge island perspective on mammalian extinctions in western North American parks. Nature 325:430-432

Newmark WD (1996) Insularization of Tanzanian Parks and the local extinction of large mammals. Conserv Biol 10: 1549-1556

Newmark WD (2008) Isolation of African protected areas. Front Ecol Environ 6:321-328

Nowak RM (ed) (1999) Walker's mammals of the world, 6th edn. Johns Hopkins University Press, Baltimore, MD

> Nyamsuren D, Joly DO, Enkhtuvshin S, Odonkhuu D, Olson KA, Draisma M, Karesh WB (2006) Exposure of Mongolian gazelles (Procapra gutturosa) to foot and mouth disease virus. J Wildl Dis 42:154-158

Ockenfels RA, Alexander A, Ticer CL, Carrel WK (1994) Home ranges, movement patterns, and habitat selection of pronghorn in central Arizona. Tech Rep 13, Ariz Game Fish Service, Phoenix, AZ

Olson KA, Fuller TD, Schaller GB, Odonkhuv D, Murray MG (2005) Estimating the population density of Mongolian gazelles Procapra gutturosa by driving long-distance transects. Oryx 39:164-169

Ottichilo WK, De Leeuw J, Skidmore AK, Prins HHT, Said MY (2000) Population trends of large non-migratory wild herbivores and livestock in the Masai Mara ecosystem, Kenya, between 1977 and 1997. Afr J Ecol 38:202-216

Ottichilo WK, de Leeuu J, Prins HTT (2001) Population trends of resident wildebeest and factors influencing them in the Masai Mara, Kenya. Biol Conserv 97:271-282

Owens MJ, Owens CD (1983) Cry of the Kalahari. Mariner Books, New York

Owen-Smith N (1987) Pleistocene extinctions: the pivotal role of megaherbivores. Paleobiology 13:351-362

Picton HD (1960) Migration patterns of the Sun River elk herd. Mont J Wildl Manag 243:279-290
Pimm SL, Ayres M, Balmford A, Branch G, Brandon K (2001) Can we defy nature's end? Science 293:2207-2208

Pulido F (2007) Phenotypic changes in spring arrival: evolution, phenotypic plasticity, effects of water and condition. Clim Res 35:5-23

Pullianen E, Sulkava S, Erkinaro E, Heikura K, Lindgren E (1983) Part VII Third International Reindeer/Caribou Symposium, Saariselk AA 23-26 August 1982. Acta Zool Fenn 175:15-16

Reading RP, Mix H, Lhagvasuren B, Tseveenmyadag N (1998) The commercial havest of wildlife in Dornod Aimag, Mongolia. J Wildl Manag 62:59-71

Reading RP, Mix HM, Lhagvasuren B, Feh C, Kane DP, Dulamtseren S, Enkhbold S (2001) Status and distribution of Kulan (Equus hemionus) in Mongolia. J Zool (Lond) 254:381-389

Robinson RA, Humphrey QPC, Learmonth JA, Maclean IMD and others (2009) Travelling through a warming world: climate change and migratory species. Endang Species Res doi: 10.3354/esr00095 (preprint 2008)

Rudd WJ, Ward AL, Irwin LL (1983) Do split hunting seasons influence elk migration from Yellowstone National Park? Wildl Soc Bull 11:328-331

Sawyer H, Lindzey F, McWhirter D, Andrews K (2002) Potential effects of oil and gas development on mule deer and pronghorn populations in western Wyoming. Trans N Am Wild Nat Resour Conf 67:350-365

Sawyer H, Lindzey F, McWhirter D (2005) Mule deer and pronghorn migration in western Wyoming. Wildl Soc Bull 33:1266-1273

Schaller G (1998) Wildlife of the Tibetan steppe. University of Chicago Press, Chicago, IL

Serneels S, Lambin EF (2001) Impact of land use change on the wildebeest migration in the northern part of the Serengeti-Mara ecosystem. J Biogeogr 28:391-407

Sinclair ARE, Norton-Griffiths M (1979) Serengeti: dynamics of an ecosystem. The University of Chicago Press, Chicago, IL

Sinclair ARE, Arcese P (1995) Serengeti II: Dynamics, management, and conservation of an ecosystem. The University of Chicago Press, Chicago, IL

Skinner MP (1925) Migration routes of elk in Yellowstone National Park. J Mammal 6:184-192

Skinner JD, Smithers RHN (1990) The mammals of the southern African subregion, 2nd edn. University of Pretoria, Pretoria

Skinner JD (1993) Springbok (Antidorcas marsupialis) treks. Trans R Soc S Afr 48:291-306

Skinner JD, Louw GN (1996) The springbok: Antidorcas marsupialis Zimmermann, 1780. Transvaal Museum Monogr 10, University of Pretoria, Pretoria

Skinner JD, Chimimba CT (2005) The mammals of the Southern African subregion, 3rd edn. Cambridge University Press, Cambridge

Smith BL, Robbins RL (1994) Migration and management of the Jackson elk herd. Research Publ. 199, US National Biological Survey, Washington, DC,

Smithers RHN (1971) The mammals of Botswana. Museum Memoir No. 4, Trustees of the National Museum of Rhodesia, Salisbury

Smuts GL (1972) Seasonal movements, migration and age determination of Burchells zebra Equus burchelli antiquorum H Smith, 1841 in the Kruger National Park. University of Pretoria, Pretoria

Spinage CA (1992) The decline of the Kalahari wildebeest. Oryx 26:147-150

Springer LM (1950) Aerial census of interstate antelope herds 
of California, Idaho, Nevada, and Oregon. J Wildl Manag 14:295-298

Swayne H (1894) Further field notes on the game animals of Somaliland. Proc Zool Soc Lond 1894:316-323

Talbot LM, Talbot MH (1963) The wildebeest in western Masailand, East Africa. Wildl Monogr 12:1-88

TAWIRI (Tanzanian Wildlife Research Institute) (2001) Aerial census of the Tarangire Ecosystem. Tanzanian Wildlife Research Institute, Arusha

Taylor RT, Martin RB (1987) Effects of veterinary fences on wildlife conservation in Zimbabwe. Environ Manag 11: 327-334

The Patagonia Times (2008) New study to explore the secrets of Chile's guanacos. 12 June 2008. Available at: www.patagoniatimes.cl/index.php/20080612551/News/ Health-Science/NEW-STUDY-TO-EXPLORE-SECRETSOF-CHILES-GUANACOS.html (accessed 18 November 2008)

Thirgood SJ, Nefdt RJ, Jeffery RCV, Kamweneshe B (1994) Population trends and current status of black lechwe in Zambia. Afr J Ecol 32:1-8

Thirgood S, Mosser A, Tham S, Hopcraft G and others (2004) Can parks protect migratory ungulates? The case of the Serengeti wildebeest. Anim Conserv 7:113-120

Thirgood S, Mlingwa C, Gereta E, Runyoro V, Malpas R, Laurenson $\mathrm{K}$, Borner M (2008) Who pays for conservation? Current and future financing scenarios for the Serengeti. In: Sinclair ARE, Packer C, Mduma SAR, Fryxell JM (eds) Serengeti III: Human impacts on ecosystem dynamics. University of Chicago Press, Chicago, IL, p 443-469

Tobey B (2003) Units 13 and 14B caribou management report. In: Healy $C$ (ed) Caribou management report of survey and inventory activities 1 July 2000-30 June 2002. Alaska Department of Fish and Game, Juneau, AK, p 108-124

Toweill DE, Thomas JE (2002) North American elk: ecology and management. Smithsonian Institution Press, Washington, $\mathrm{DC}$

Ulvevadet B, Klokov K (2004) Family-based reindeer herding and hunting economies, and the status and management of wild reindeer/caribou populations. University of Tromsø

van Aarde RJ, Jackson TP (2007) Megaparks for metapopulations: Addressing the causes of locally high elephant num-

Editorial responsibility: Brendan Godley,

University of Exeter, Cornwall Campus, UK bers in southern Africa. Biol Conserv 134:289-297

> Van Vuren D, Bray MP (1986) Population dynamics of bison in the Henry Mountains, Utah. J Mammal 67:503-511

Verlinden A (1998) Seasonal movement patterns of some ungulates in the Kalahari ecosystem of Botswana between 1990 and 1995. Afr J Ecol 36:117-128

Vitousek PM, Ehrlich PR, Ehrlich AH, Matson PA (1986) Human appropriation of the products of photosynthesis. BioScience 36:368-373

Walther FR (1972) Social grouping in Grant's gazelle (Gazella granti Brooke, 1872) in the Serengeti National Park. Z Tierpsychol 31:348-403

WDPA (World Database on Protected Areas) Consortium (2005) World Database on Protected Areas. Copyright World Conservation Union IUCN and UNEP-World Conservation Monitoring Centre UNEP-WCMC. www.unepwcmc.org/wdpa/ (accessed 7 Aug 2005)

> Wilcove DS, Wikelski M (2008) Going, going, gone: Is animal migration disappearing? PLoS Biol 6:e188

Williams S (2002) Status and action plan for Grevy's zebra. In: Moehlman PD (ed) Equids: zebras, asses, and horses status survey and conservation action plan. IUCN/Species Survival Commission Equid Specialist Group, IUCN, Gland

Williamson DT, Mbano B (1988) Wildebeest mortality during 1983 at Lake Xau, Botswana. Afr J Ecol 26:341-344

Williamson D, Williamson J, Ngwamotsoko KT (1988) Wildebeest migration in the Kalahari. Afr J Ecol 26:269-280

Wingard JR, Zahler P (2006) Silent steppe: the illegal wildlife trade crisis in Mongolia. Mongolia Discussion Papers, East Asia and Pacific Environment and Social Development Department, The World Bank, Washington, DC

Whyte IJ, Joubert SCJ (1988) Blue wildebeest population trends in the Kruger National Park and the effect of fencing. S Afr J Wildl Res 18:78-87

Yoakum JD (1978) Pronghorn. In Schimdt JL, Gilbert DL (eds) Big game of North America: ecology and management. Wildlife Management Institute, Washington, DC, and Stackpole Books, Harrisburg, PA, p 103-121

Yoshihara Y, Ito TY, Lhagvasuren B, Takatsuki S (2008) A comparison of food resources used by Mongolian gazelles and sympatric livestock in three areas in Mongolia. J Arid Environ 72:48-55

Submitted: May 20, 2008; Accepted: November 21, 2008 Proofs received from author(s): April 6, 2009 